\title{
High Speed Rotary System for Catheter Based 3-D Imaging with Optical Coherence Tomography (OCT)
}

\author{
by \\ Antonio Mauro, B.Eng. \\ Ryerson University \\ Toronto, Ontario, Canada, 2005
}

\author{
A thesis \\ presented to Ryerson University \\ in partial fulfillment of the \\ requirements for the degree of \\ Masters of Science \\ in the program of \\ Biomedical Physics
}

Toronto, Ontario, Canada, 2009

C Antonio Mauro 2009 


\section{AUTHOR'S DECLARATION}

I, Antonio Mauro, hereby declare that I am the sole author of this thesis.

I authorize Ryerson University to lend this thesis to other institutions or individuals for the purpose of scholarly research.

Signature

I further authorize Ryerson University to reproduce this thesis by photocopying or by other means, in total or in part, at the request of other institutions or individuals for the purpose of scholarly research.

Signature 


\section{BORROWER'S PAGE}




\title{
ABSTRACT
}

\section{High Speed Rotary System for Catheter Based 3-D Imaging with Optical Coherence Tomography (OCT)}

\author{
Antonio Mauro, Master of Science, 2009 \\ Department of Physics, Program of Biomedical Physics, Ryerson University
}

Optical Coherence Tomography (OCT) is an addition to the other tomographic imaging techniques of $\mathrm{x}$-ray computed tomography, magnetic resonance imaging, and ultrasound imaging. OCT uses optical reflections of biological tissues as opposed to $\mathrm{x}$-rays, RF fields, and sound waves to obtain images. A rotary and pullback system has been developed for use with OCT. The system was developed to facilitate the three dimensional imaging of various lumens in humans and animals. The system is capable of rotating at a rate of $200 \mathrm{~Hz}$. At this rate the rotary system will allow for a frame acquisition rate of $200 \mathrm{fps}$ which is significantly higher than the highest published acquisition rate to date of 108 fps. The probes used with the system were modeled after the Intravascular Ultrasound (IVUS) miniature torque cable design. The probes can be sealed and sterilized between subjects without being damaged; unlike the single use IVUS probes. The rotary system was used to image the outer ear of a mouse in vivo. A lateral slice from the resulting three dimensional image was compared to the general histology of a mouse ear. The image compared well to the general anatomy as found on histology. 


\section{ACKNOWLEDGEMENTS}

First of all I would like to thank my supervisor, Dr. Victor Yang, for giving me the opportunity to be part of his OCT group and allowing me to work with and interact with many wonderful and gifted people. Working with Victor has been a great inspiration. His unyielding dedication to everything that he does in life sets a prime example for all of us in his group to follow. Thanks Victor, not only for your guidance with matters related to my project, but also for being a good person to all of your students.

I would also like to thank my co-supervisor, Dr. Michael Kolios, for guiding me through key points over the past couple of years. Mike is also a man of unyielding dedication and a great inspiration. I would like to thank Mike for often taking time to meet with me to discuss my project and my approach to accomplishing certain things over the last 2 years.

I would like to thank Dr. Xijia Gu. Dr. Gu was an important part of my supervisory committee who always had excellent advice and helped me through the tough task of creating optics for the rotary probes. Dr. Gu is another man with a superior dedication and an inspiring demeanor. Dr. Gu is an example of how a man can be calm and cool while putting forth good work. Thanks Dr. Gu!

My whole experience over the last couple of years would not have been a possibility if it was not for Dr. Pedro Goldman, Dr. Carl Kumaradas, Dr. Juliana Carvalho, and Mary "the mother hen" Neelands allowing me to transfer into the Biomedical Physics program.

Being part of this program has been a great experience. The dedication the physics 
department puts towards making the student experience as healthy as possible is a prime example for all departments to follow.

I am also grateful to Dr. Alex Vitkin and Dr. Brian Wilson for taking me into the Wilson Group at Princess Margaret Hospital as one of their own. If it was not for the unlimited access to all of the resources available to me at PMH I would not have been able to complete this project. I would also like to thank Alex and Brian for having an open door policy and offering guidance whenever possible.

Now on to the other students in the OCT group. I would like to thank the PhD students, Dr. Beau Standish, Dr. Nigel Munce, and PhD candidate Adrian Mariampillai for their guidance and assistance with whatever queries or questions I had. I would like to thank my fellow masters students Mike Leung and Mark Harduar. Thanks Mike, for being a good lab mate and sharing interesting OCT and even more interesting non-OCT conversations with me (and for letting me borrow one of your window chambered mice). Thanks Mark, if it was not for your hclp with some coding issues this project would have taken much longer to complete. I also want to thank one of our summer students, Louis Tan, for helping out with some coding (kecp up the good work Louis!) and our not so summer students Ken and Tim for making things interesting and humbling us with their intelligence.

Thanks to Jenny for making everything in our new Ryerson lab run so smoothly (and also for assuring us that Victor is a human that eats and sleeps like the rest of us). Thanks to 
Tess and Sally for making everything in the physics department run as smoothly as possible and helping us plan extracurricular events. Thanks to Iosif for keeping the graduate assistants in order and for putting up with my antics. You are a good man Mr. Iosif! Thanks to Leo and Matt for help with my crazy Macbook and retrieving my data when the hard drive crashed. Without your help, I would have been finishing this degree at a much later date. Thanks to Eva and Barb for making everything at PMH run smoothly. Thanks to Rodney Martin of the PMH machine shop for doing a great job of machining the parts for my rotary system. Thanks to Maria Cirrocco for allowing me to put my probes through a cleaning cycle at St. Michael's Hospital and for allowing me to converse with and shadow the doctors in the endoscopy unit. My time at St. Mike's was an enlightening and memorable experience.

This work was funded by the Canadian Institutes of Health Research (CIHR). 


\section{DEDICATION}

To my father and mother (Michele and Maria) for their unconditional love and support. 


\section{TABLE OF CONTENTS}

AUTHOR'S DECLARATION ....................................................................................... ii

BORROWER'S PAGE........................................................................................................

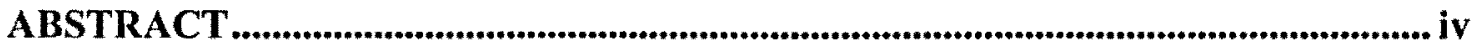

ACKNOWLEDGEMENTS .............................................................................................. v

DEDICATION............................................................................................................................ viii

TABLE OF CONTENTS ...................................................................................................... ix

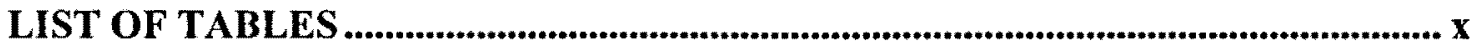

LIST OF FIGURES .............................................................................................................. xi

CHAPTER 1: INTRODUCTION AND BACKGROUND....................................... 1 -

1.1. Optical Coherence Tomography (OCT) ……............................................. 1 -

1.2. Spectral Domain OCT (SD-OCT) ……........................................................ 7 -

1.3. OCT in Cardiology .................................................................................. 11 -

1.4. Objective ................................................................................................ 13 -

CHAPTER 2: ROTARY SYSTEM DESIGN ............................................................- 14 -

2.1. Main System Design .................................................................................. 14 -

2.2. Probe Design ........................................................................................... 24 -

2.2.1. Catheters …….........................................................................................-24 -

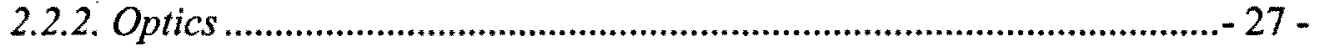

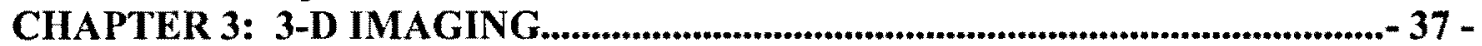

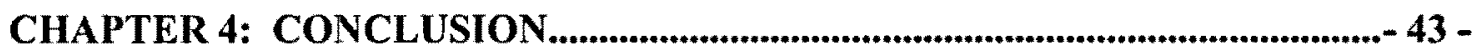

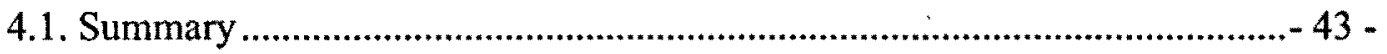

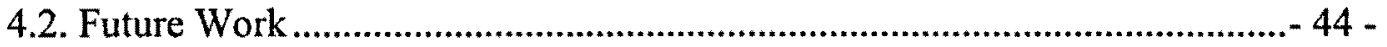

4.2.1. Doppler …..................................................................................... 44 -

4.2.2. Mouse Aorta Imaging ……………................................................. 46 -

REFERENCES...................................................................................................... 51 -

APPENDIX: MATLAB CODE...................................................... 54 - 


\section{LIST OF TABLES}

Table 2.1 - Frequency swept laser power $15-$

Table 2.2 - Power through FORJ with tuning on ......................................................... 15 Table 2.3 - Power through FORJ with tuning off. $15-$ Table 2.4-Combinations of various CL spacer and GRIN lengths and the resulting working distance. $-34-$ 


\section{LIST OF FIGURES}

Figure 1.1 - Basic setup of a Michelson interferometer................................................ - 2 -

Figure 1.2 - In vivo OCT image, with micrometer resolution, of the structures

surrounding the fingernail on one of the author's baby fingers. $-3-$

Figure 1.3 - Spectral distribution and corresponding interferogram for different light sources. $-4-$

Figure 1.4 - Theoretical interferogram for a broadband light source with a central wavelength of $1.3 \mu \mathrm{m}$ and a $60 \mathrm{~nm}$ bandwidth in tissue with an index of refraction of $1.4 \ldots$

Figure 1.5 - Illustration of transverse resolution, $\Delta x$, and depth of focus, $b$, for a beam focused with an optical lens..... $5-$ Figure 1.6 - The number of publications per year with optical coherence tomography in the title as indexed in PubMed and Scitation. $-7-$ Figure 1.7 - Basic configuration of optical frequency domain reflectometry (OFDR). 8 Figure 1.8 - Schematic of the Frequency Domain Mode Locking (FDML) capable SSOCT system used for imaging $-10-$

Figure 1.9 - Intravascular imaging of a fibrous coronary plaque showing: $i$-intimia (with intimial hyperplasia), $m$ - media, and $a$-adventitia... $-12-$ Figure 2.1 - CAD drawing of the first iteration of the rotary pullback system design- 14 Figure 2.2 - 3-D CAD drawing of the fiber optic rotary joint (FORJ) .......................... 15 Figure 2.3 - Data from Table 2.2 in graphical form.............................. 16 Figure 2.4 - 3-D CAD drawing of the rotary motor showing an isometric view ........- 17 Figure 2.5 - Left: Example of a FC-APC connector. Right: Example of a SC-APC

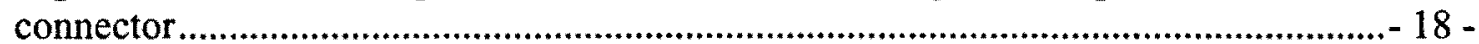
Figure 2.6 - Side and isometric views of the catheter support holding an outer

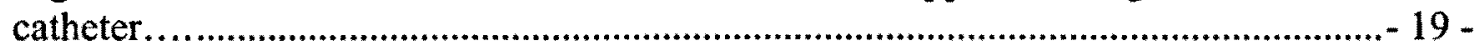
Figure 2.7 - Distal coupling of the first prototype of the rotary pullback system.......-20 Figure 2.8 - First prototype of the rotary pullback system............................................ 20 Figure 2.9 - Image of a thumb and index finger grasping a radial probe from

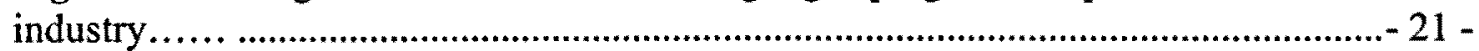
Figure 2.10 - Distal coupling of the current rotary pullback system prototype. .......... 22 Figure 2.11 - Current Rotary System Prototype............................................................... 23 Figure 2.12 - Left: 2-D image of a thumb (bottom of image) and index finger (top of image) grasping a catheter. Right: 2-D image of a probe taped to an IR card.............. 24 Figure 2.13 - Imaging end of an Intravascular Ultrasound probe. ................................ 25 Figure 2.14 - Left: Adaptor end of an outer catheter. Right: Adaptor with cap........... 26 Figure 2.15 - Completed probe ready for sterilization. ................................................... 26 Figure 2.16 - Polished probe tip in the midst of polishing glue................................... 28 Figure 2.17 - Radial image of a probe with $0.45 \mathrm{~mm}$ GRIN optics taped to an IR card..... - $29-$

Figure 2.18 - Optical profiler results for the polished probe surface at an effective magnification of about $10 \mathrm{x}$. $-30-$ Figure 2.19-Optical profiler results for the polished probe surface at an effective magnification of about $50 \mathrm{x}$. $-30-$ 
Figure 2.20 - SEM results of polished probe surface at a magnification of $300 x$ (left) and a section of the polished surface at $10000 \mathrm{x}$ (right)

Figure 2.21 - Sketch showing the light path through the optics of a probe with a corcless

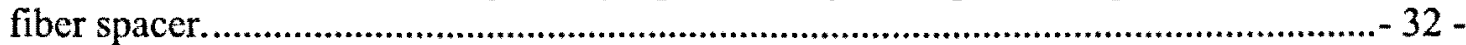

Figure 2.22 - Power profile for coreless spacer probe characterization............. - 33 Figure 2.23 - Titanium dioxide phantom imaged to test the quality of a probe at the forward-looking stage.

Figure 2.24 - Probe optics with CL spacer magnificd 100x viewed from two different angles

Figure 2.25 - Probe with CL Spacer taped to an IR card. .............................................-36 -

Figure 3.1 - Window chambered mouse with the imaged ear.......................................- 39 -

Figure 3.2 - 3-D image of a radial scan of a mouse ear. ...............................................- 39 -

Figure 3.3 - Axial cross sections of the mouse ear image..............................................- 40 -

Figure 3.4 - Lateral cross sections of the mouse ear image. . .......................................- 41 -

Figure 3.5 - Cross section of the mouse ear scan with a radial probe compared to the galvo scanner results and histology.

Figure 4.1 - Left: A structural frame of the mouse ear. Right: Corresponding Doppler image.

Figure 4.2 - Three dimensional SS-OCT image of an ex vivo mouse aorta $-47-$

Figure 4.3 - Three dimensional SS-OCT image of the ascending aorta portion of an ex

vivo mouse aorta.. ....................................................................................................... 47

Figure 4.4 - Latcral cross section of the descending aorta portion of Figure 4.2........ 48 -

Figure 4.5 - Axial cross sections of the mouse aorta.................................................... 48 -

Figure 4.6-Left: Axial cross section of the bifurcation of the ascending aorta from

Figure 4.3 with the aortic arch on the right and the brachiocephalic artery on the left.

Right: Lateral cross section of the brachiocephalic artery..

Figure 4.7 - In vivo images of aortic blood flow in a rat using transesophageal Doppler

OCT methods. 


\section{CHAPTER 1: INTRODUCTION AND BACKGROUND}

This thesis describes the design and construction of a rotary pullback system that was used with the relatively new imaging modality of Optical Coherence Tomography (OCT). This thesis shows the potential of the rotary pullback system to create three dimensional reconstructions of tissue surrounding various lumens in animals and humans.

\subsection{Optical Coherence Tomography (OCT)}

Optical Coherence Tomography (OCT) was first introduced to the scientific community in 1991 by a group at the Massachusetts Institute of Technology (MIT) headed by Dr. James G. Fujimoto [1] as an addition to the other tomographic imaging techniques of $\mathrm{x}$-ray computed tomography, magnetic resonance imaging, and ultrasound imaging. OCT uses optical reflections of biological tissues as opposed to $\mathrm{x}$-rays, $\mathrm{RF}$ fields, and sound waves to obtain images. The most common setup used in OCT is modeled after the Michelson interferometer. A Michelson interferometer consists of a coherent light source, half-silvered mirror (or beam splitter), light detector, and two reflecting mirrors. Figure 1.1 shows the basic setup of a Michelson interferometer. 


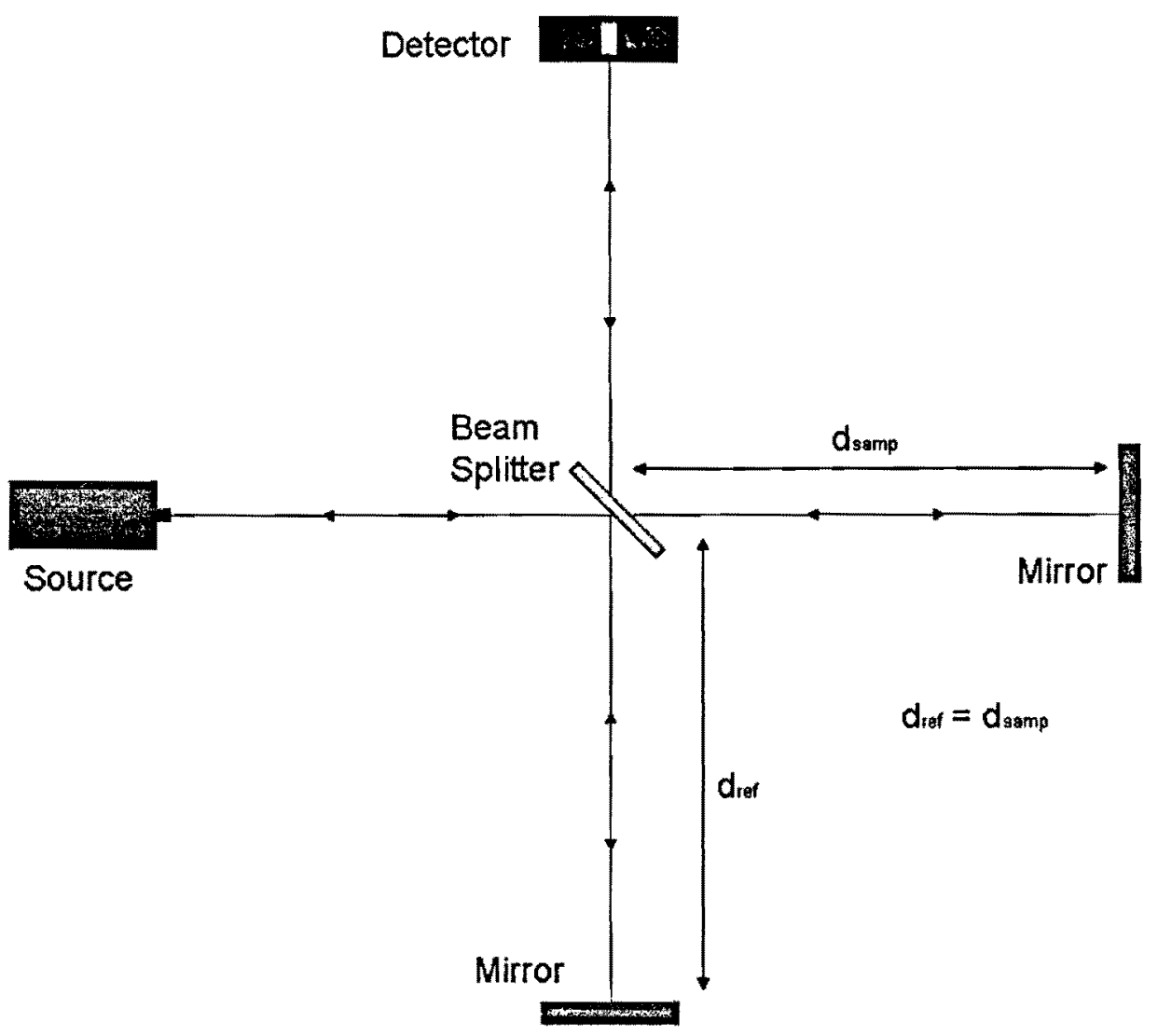

Figure 1.1-Basic setup of a Michelson interferometer.

In time domain OCT (TD-OCT) the light source is a broadband partially coherent light source and one mirror is replaced by a tissue sample. Once the light leaves the source it enters a beam splitter which directs half of the light to the sample and half to the mirror. The intensity of the beam going to the detector from the beam splitter depends on the difference in path length between the reference and sample arm, the amount of light reflected off the sample and the reference mirror, the spectral distribution of the beam, and the coherence length of the light. By rapidly decreasing and increasing the reference arm distance, the coherence gate of the source moves along the depth of the sample producing an axial scan or A-scan. If the sample arm is translated laterally a 2-D image, B-scan, can be created. This will provide a structural image of the sample. An example 
of this can be seen in Figure 1.2. This figure reveals the structures surrounding a human fingernail in vivo. It clearly shows the fingernail root, the skin above the root, and the cuticle. The figure also shows a shadow caused by the cuticle, which readily scatters light.

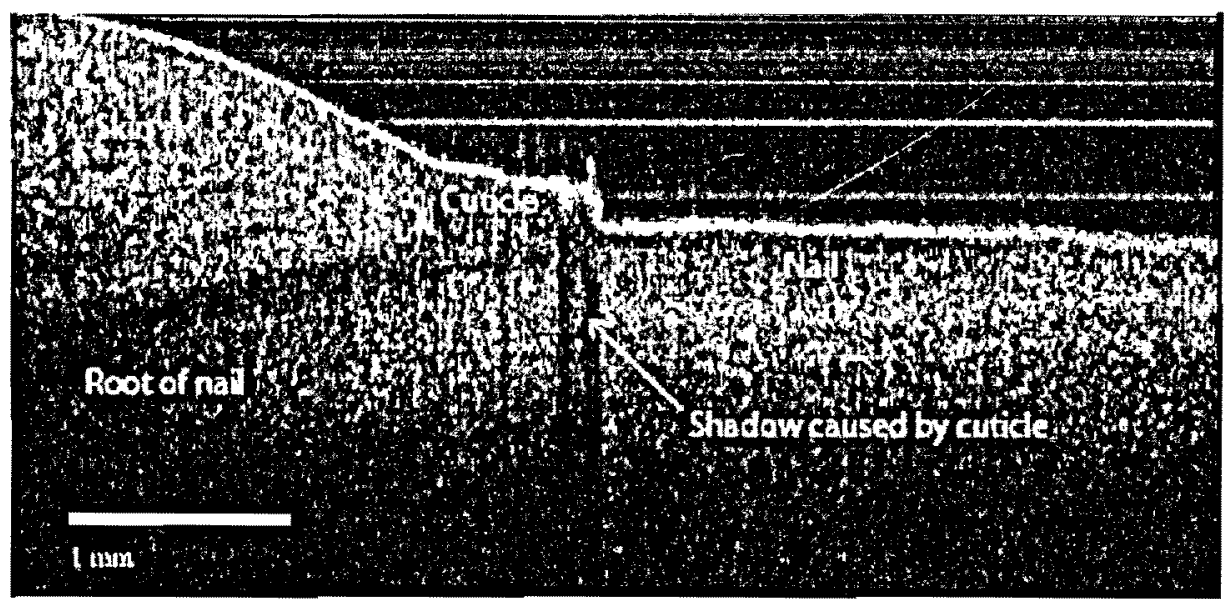

Figure 1.2 - In vivo OCT image, with micrometer resolution, of the structures surrounding the fingernail on one of the author's baby fingers.

Plotting the intensity of the beam reaching the detector as a function of the position of the reference mirror will result in an interferogram. The spectral distribution of the light source will affect the pattern of the interferogram. Figure 1.3 shows how the spectral distribution of the light source influences the envelope of the interferogram. A broad spectrum results in a narrow envelope and a narrow spectrum results in a broad envelope. The envelope also contains information regarding the time coherence of the light source. The mathematical relation between the spectrum and the envelope is a Fourier transform. 


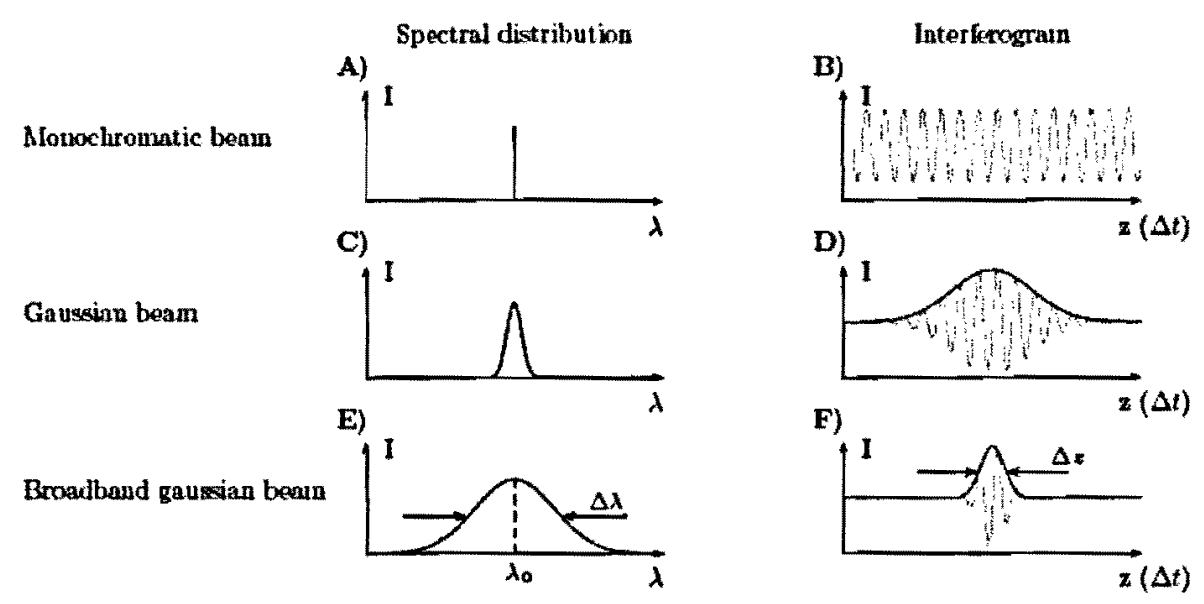

Figure 1.3 - Spectral distribution and corresponding interferogram for different light sources. All units are arbitrary. ( $\Delta \lambda=$ source bandwidth, $\Delta z=$ coherence length [2]

The bandwidth, $\Delta \lambda$, of a source is typically defined at the full-width-half-max (FWHM) (ie. $3 \mathrm{~dB}$ bandwidth) of the Gaussian spectrum. The bandwidth, of a source limits the interference pattern to a range called the coherence length ( $\Delta \mathrm{z}$ or commonly $l_{c}$ ), which is defined as:

$$
\Delta z=l_{c}=\frac{2 \ln (2)}{\pi \cdot n} \frac{\lambda_{0}^{2}}{\Delta \lambda}
$$

where, $\lambda_{0}$ is the central wavelength of the source and $n$ is the index of refraction of the sample. Figure 1.4 is a theoretical interferogram for a near infrared light source with a central wavelength of $1.3 \mu \mathrm{m}$ and a $60 \mathrm{~nm}$ bandwidth. Assuming a tissue index of refraction of 1.4 , the coherence length comes to be $8.9 \mu \mathrm{m}$. 


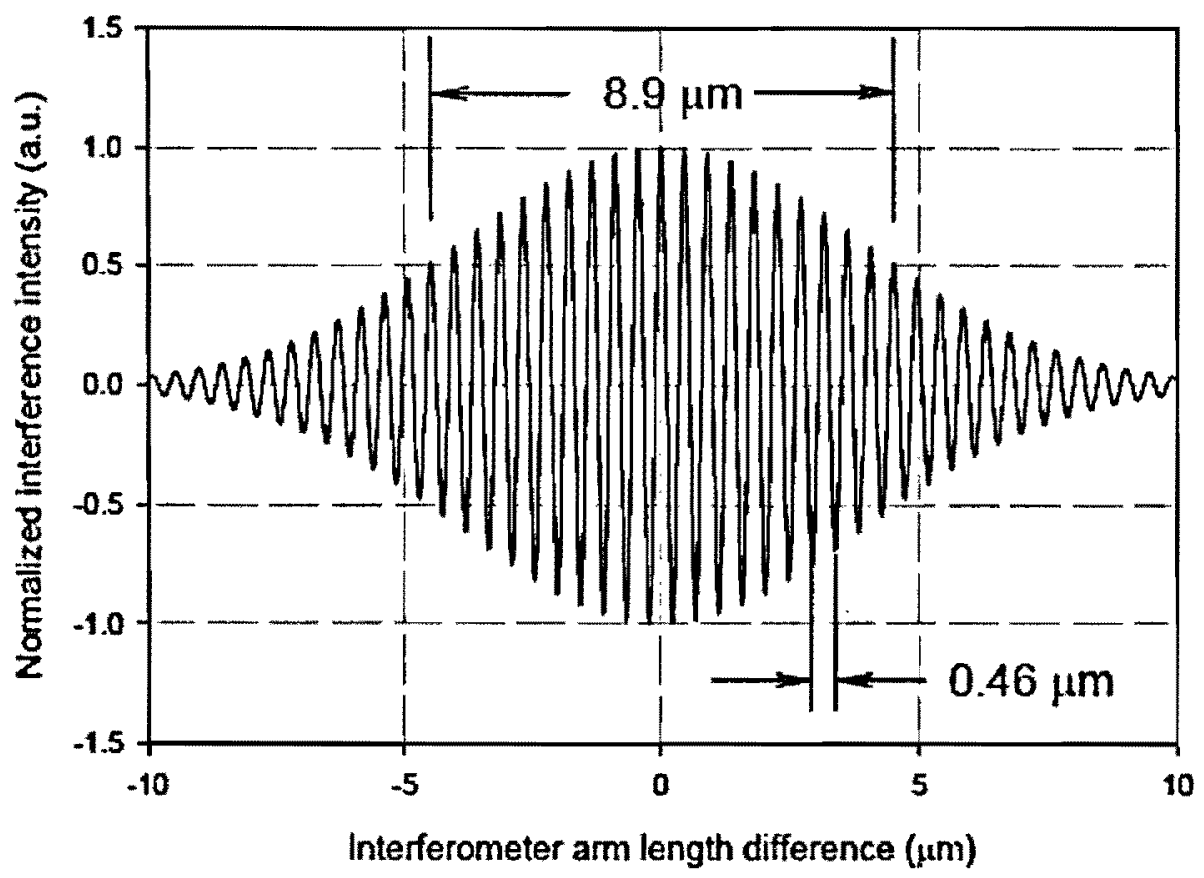

Figure 1.4 - Theoretical interferogram for a broadband light source with a central wavelength of $1.3 \mu \mathrm{m}$ and $a 60 \mathrm{~mm}$ bandwidth in tissue with an index of refraction of 1.4. [3]

The transverse resolution (ie. horizontal resolution) is mainly determined by the optics in the sample arm. In a typical OCT system the laser light is first collimated and then focused onto a sample using an optical lens. The beam waist of the focused beam defines the transverse resolution, and the Rayleigh range defines the depth of focus. If a lens of focal length " $f$ ' is used, then the transverse resolution, $\Delta x$, and depth of focus (ie, twice the Rayleigh range), $b$, can be defined as:

$$
\begin{gathered}
\Delta x=\frac{4 \lambda_{0}}{\pi} \frac{f}{d} \\
b=\frac{\pi \Delta x^{2}}{2 \lambda_{0}}
\end{gathered}
$$

where, $\mathrm{d}$ is the 1/e beam size of the collimated beam at the optical lens. Figure 1.5 is a visual representation of the aforementioned parameters. 


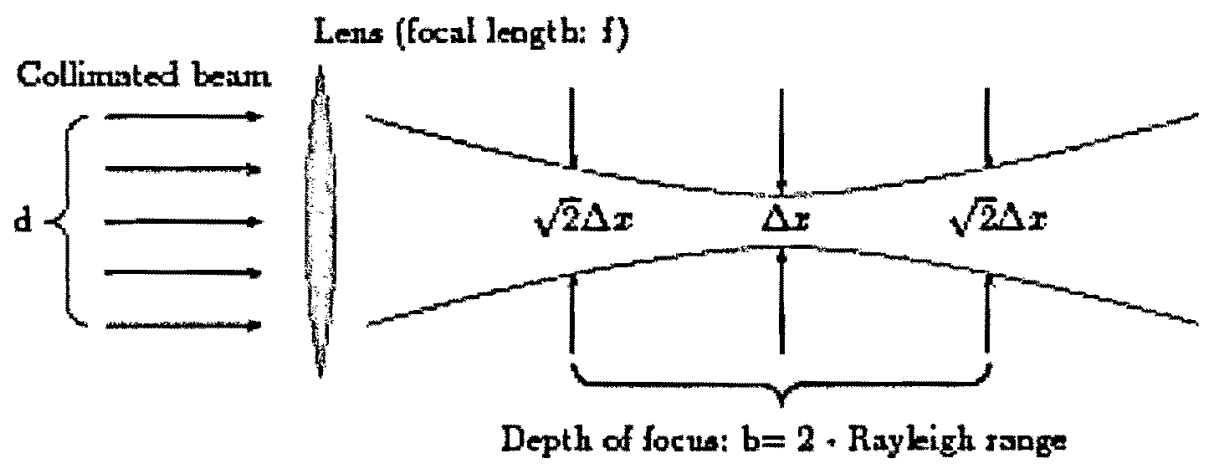

Figure 1.5 - Illustration of transverse resolution, $\Delta x$, and depth of focus, $b$, for a beam focused with an optical lens. [2]

OCT has proven to be advantageous because of its high spatial resolution $(\sim 1-$ $10 \mu \mathrm{m})[4]$. Another advantage of OCT is that its sample arm can be packaged into fiber optic cables with very small diameters allowing for the biological investigation of several tissue types such as: heart tissue $[4,6]$, microvessels [5], esophageal tissue $[6,7]$, and brain tissue $[8,9]$, just to mention a few. This is possible because the single mode fiber used to transport the light to the tissue is typically no larger than $250 \mu \mathrm{m}$ in diameter.

OCT has experienced rapid development since it was first introduced. This is due to a growing interest in the field and the potential impact on clinical medicine. This is evident in the increase of publications from 200 peer reviewed journal publications in the year 2000 to 800 publications in 2006 and a total of over 4000 publications to date [10]. The following figure shows the yearly growth of OCT scientific and medical research publications indexed by two popular databases, PubMed and Scitation. 


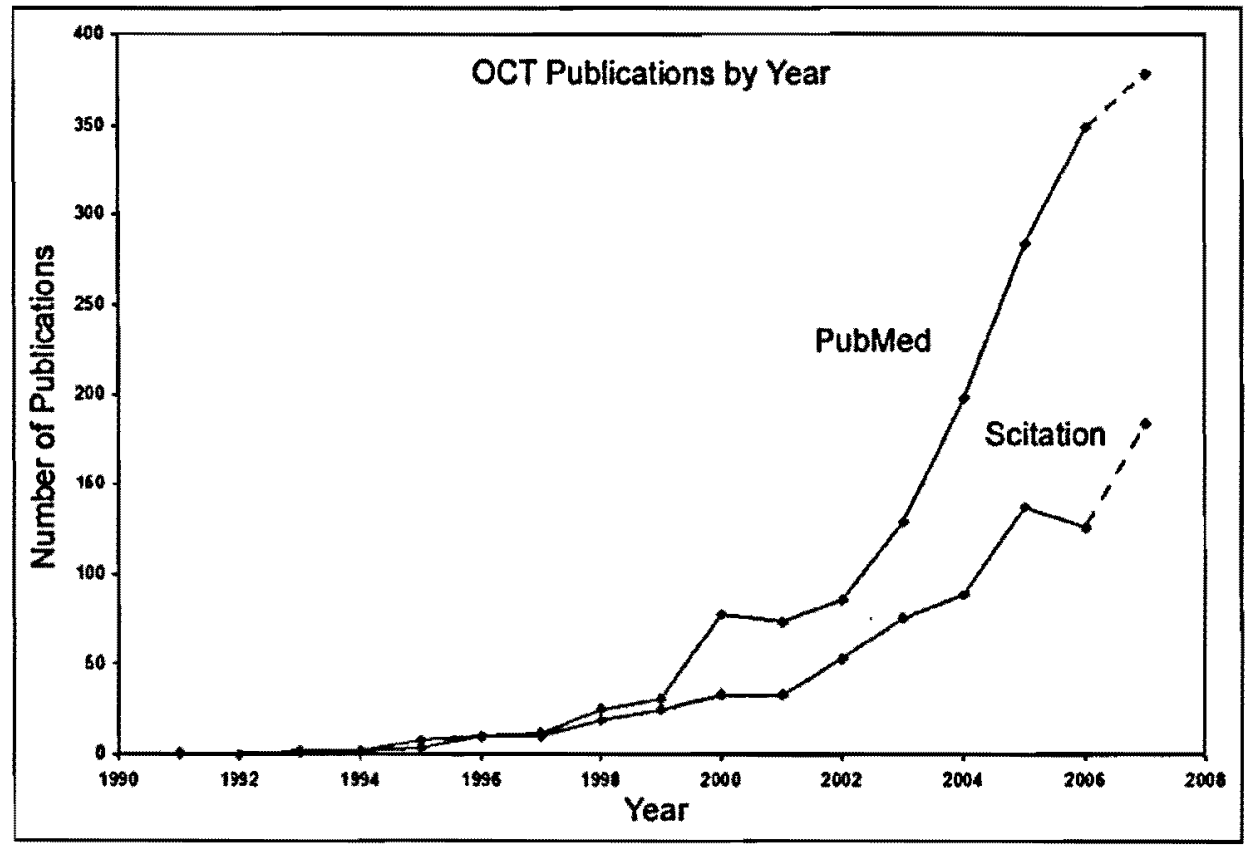

Figure 1.6 - The number of publications per year with optical coherence tomography in the title as indexed in PubMed and Scitation. Data for 2007 was extrapolated based on the numbers up to September 2007 [11].

\subsection{Spectral Domain OCT (SD-OCT)}

In SD-OCT the reference arm typically does not move and a Fourier relation is exploited where the depth scan is immediately calculated by a Fourier-transform of the acquired spectra. The basis of SD-OCT is optical frequency domain reflectometry (OFDR) [12]. Figure 1.7 shows the basic configuration of OFDR using a tunable light source and a fiber-optic interferometer. 


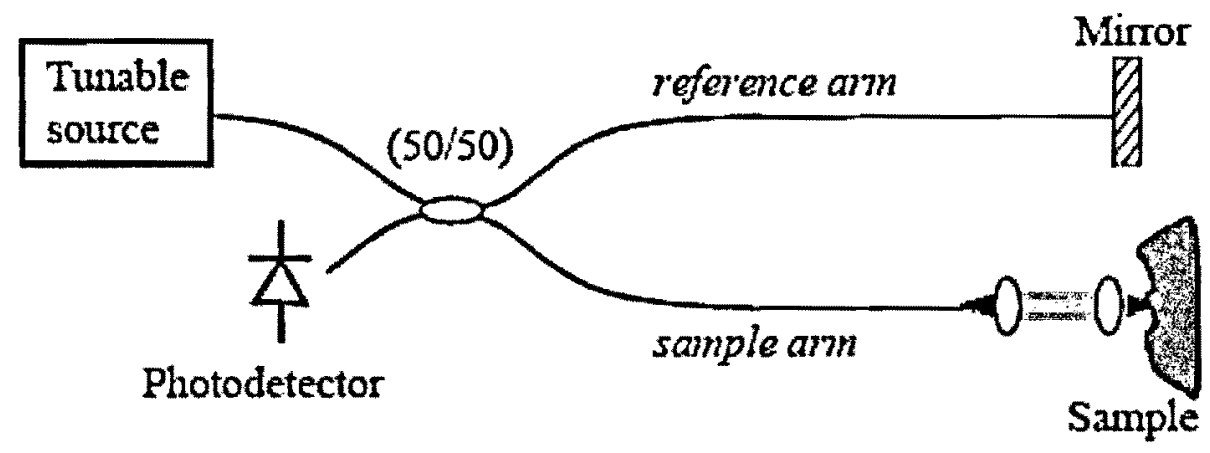

Figure 1.7 - Basic configuration of optical frequency domain reflectometry (OFDR). [12]

The source output is split into a reference arm and a sample arm that illuminates and receives light reflected from the sample. A photo detector detects the interference between the reference arm and sample arm light. Typically the wavelength of the monochromatic light source is swept and the path lengths of the reference arm and sample arm are held constant. The A-line profile is obtained by a discrete Fourier (DFT) of the sampled detector signals.

SD-OCT techniques include Fourier Domain OCT (FD-OCT) and swept source OCT (SS-OCT). FD-OCT uses a broadband laser source with a diffraction grating and a photodiode array in the detection arm. SS-OCT uses a rapidly tunable, narrow-linewidth laser source over a broad optical bandwidth with a single photodiode in the detection arm. While FD-OCT and SS-OCT have similar sensitivity advantages over conventional TD-OCT systems, the SS-OCT system can detect interference fringes over a substantially longer range of time delays between reflections from reference and sample interfaces. The system used for the work done in this thesis was a SS-OCT system. For this reason, any OCT system mentioned or described from this point on will be a SS-OCT system.

In SS-OCT the Fourier transform of the light reflected from the sample provides a 
back-reflection profile as a function of depth. The depth information is obtained by the different interference profiles obtained from the difference in sample and reference path lengths. Larger differences in the optical path length between the sample and the reference arm result in interference signals with higher frequencies [13].

The light intensity perturbation at the exit of the interferometer (ie. moving from the sample towards the detector) can be described by:

$$
I_{D}=I_{R}+I_{S}+\frac{1}{2} \int_{0}^{z} \int_{0}^{\infty} G(f) p\left(z^{\prime}\right) p_{R} \cos \left\{\frac{4 \pi f}{c}\left[n_{S}(f) z^{\prime}-z_{R}\right]\right\} d f d z^{\prime}
$$

where, $I_{R}$ corresponds to the intensity of the reflected reference beam, $I_{S}$ is the total intensity of the returned sample beam, in which all the scatters contribute, and the third term is the mutual interference of all scattering waves (called the autocorrelation function of scattering or a parasitic term $[13,14,15])$. The origin of coordinates in the sample arm, $\mathrm{O}_{\mathrm{S}}$, is usually set at the sample surface. The origin of coordinates in the reference arm, $\mathrm{O}_{R}$, is at a point where the optical group delay to the coupler matches that between $\mathrm{O}_{\mathrm{S}}$ and the coupler of the sample arm. In equation (4), $\mathrm{p}(\mathrm{z})$ represents the backscattering coefficient distribution in the sample, where $\mathrm{z}$ is measured from $\mathrm{O}_{\mathrm{s}}$. The mirror position is $z_{R}$ measured from $O_{R}$. The spectral intensity distribution of the light source in terms of the total positive frequency is $G(f)$. Assuming no dispersion, $n_{S}(f)$ represents the refractive index of the sample. Finally, $\mathrm{c}$ is the velocity of light in a vacuum.

The spectral interferogram can be obtained at the exit of the interferometer with a single detector when using a swept/tunable laser source instead of a wide band source. From equation (4), assuming an ideal spectrum is described by the detector; the spectral interferogrm can be described as: 


$$
I_{D}(f)=\frac{1}{4} G(f)\left\{p_{R}^{2}+\int_{o}^{z} p_{S}^{2}\left(z^{\prime}\right) d z^{\prime}+2 \operatorname{Re}\left[\int_{o}^{z} p\left(z^{\prime}\right) p_{R} e^{j 4 \pi \frac{f}{[}\left[n_{S}\left(f_{o}\right) z^{\prime}-z_{R}\right]} d z^{\prime}\right]\right\}
$$

This can also be written in terms of the wave number, $\mathrm{k}$ :

$$
I(k)=\frac{1}{4} G(k)\left\{p_{R}^{2}+\int_{0}^{z} p_{S}^{2}\left(z^{\prime}\right) d z^{\prime}+2 \operatorname{Re}\left[\int_{0}^{2} p\left(z^{\prime}\right) p_{R} e^{j 4 \pi k\left\{n_{S}\left(k_{o}\right) z^{\prime}-z_{R}\right]} d z^{\prime}\right]\right\}
$$

In SD-OCT (ie. FD-OCT and SS-OCT), the spectral interferogram of a single reflector located at $z_{\mathrm{o}}$ relative to $\mathrm{O}_{\mathrm{S}}$ can be expressed as:

$$
I_{D}(k)=\frac{1}{4} G(k)\left\{p_{R}^{2}+p^{2}+2 p p_{R} \cos \left[4 \pi\left(z_{o}-z_{R}\right) k\right]\right\}
$$

where, $z_{R}$ is once again the mirror position relative to $O_{R}$ and $p$ is defined as the backscattering coefficient of the single scatter [13].

Figure 1.8 is a schematic of the SS-OCT system used for the imaging presented in this thesis.

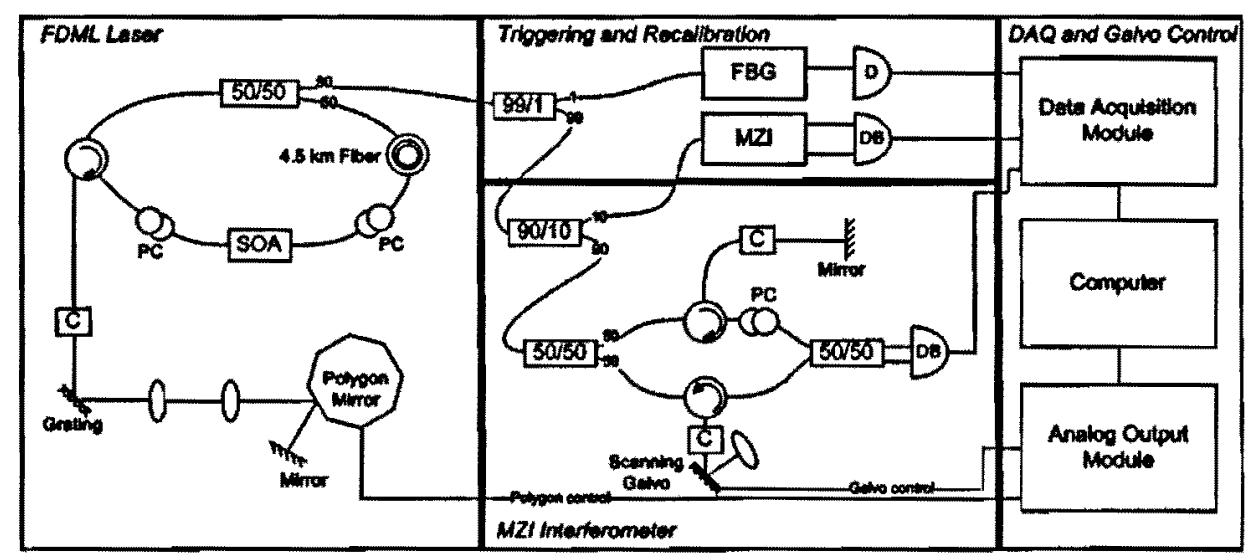

Figure 1.8 - Schematic of the Frequency Domain Mode Locking (FDML) capable SS-OCT system used for imaging. SOA: Semiconductor optical amplifier, PC: Polarization controller, C: Collimator, FBG: Fiber Bragg grating, MZI: Mach-Zehnder interferometer, D: Detector, DB: Dual balanced detector [16]

The SS-OCT system uses a 36-67 kHz Frequency Domain Mode Locked (FDML) fiberring laser incorporating a polygon-based tunable filter. The bandwidth (ie. tuning range) 
of the laser is $112 \mathrm{~nm}$ centered at $1310 \mathrm{~nm}$. The average output power of the laser is 48mW. A fiber Bragg grating provides the A-scan trigger and a Mach-Zehnder interferometer $(\mathrm{MZI})$ is used as opposed to a Michelson interferometer. In a MZI configuration the signal goes through a beam splitter before it enters the detector and $100 \%$ of the signal goes to one channel and $0 \%$ of the signal goes to the other channel. The MZI is used to reduce the noise in the images by comparing the noise in the channel without a signal to the one with a signal. The scanning galvo in Figure 1.8 is an OCT probe which scans a tissue sample externally with a raster scan type pattern in order to get a 3-D image. When the system is used with a hand-held probe or a rotary probe, the scanning galvo in Figure 1.8 is replaced with the appropriate probe.

\subsection{OCT in Cardiology}

OCT has been applied extensively in the field of cardiology in the past decade. It was first used to examine the structural integrity of coronary artery vasculature. OCT has become useful in imaging cardiovasculature due to several technological advances, such as the development of rotational catheter based probes, acquisition speed improvement, and the introduction of functional OCT modalities. In vivo imaging and validation have been performed as a result of these advances [17].

Structural OCT penetrates about $1-3 \mathrm{~mm}$ into tissue and has been compared to intravascular ultrasound (IVUS) images (the current standard in intracoronary imaging). OCT provides a higher resolution by about an order of magnitude when compared to IVUS $[17,18]$. The superior resolution of OCT is demonstrated in Figure 1.9. 

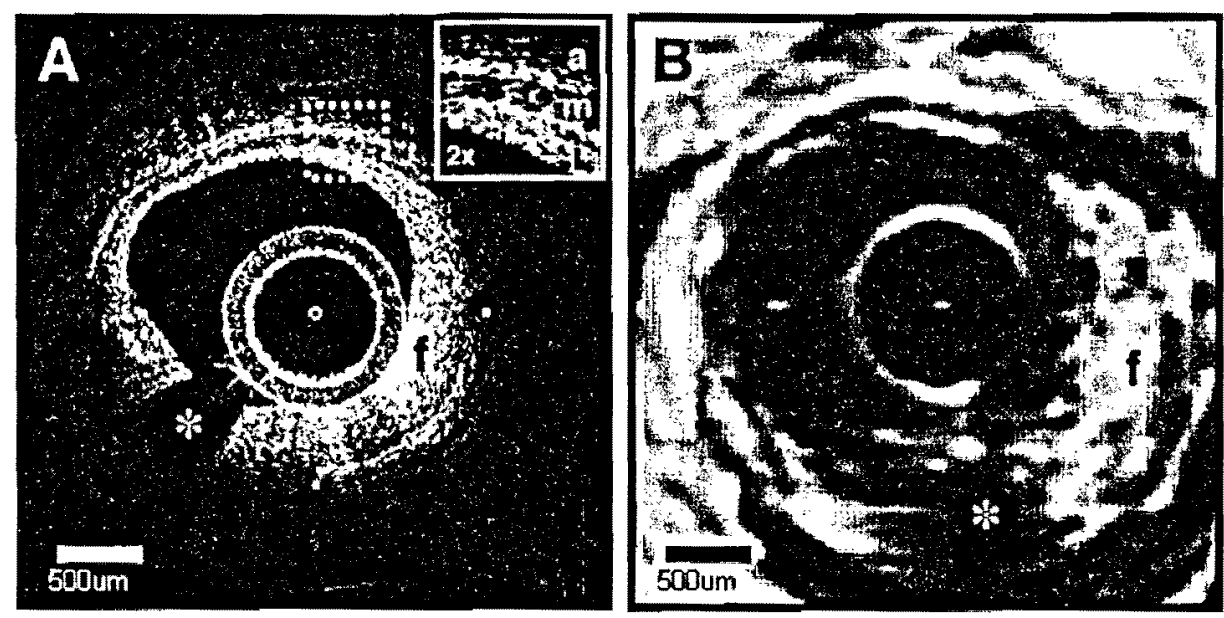

Figure 1.9 - Intravascular imaging of a fibrous coronary plaque showing: $i$ - intimia (with intimial hyperplasia), $m$-media, and $a$-adventitia. The left figure is an intravascular OCT image showing a clear delineation of layers, including the internal and external elastic laminea. A fibrous plaque $(f)$ is visible and partially obscured by a guidewire shadow artifact $\left({ }^{*}\right)$. The right figure is a corresponding 30 -Mllz IVUS image at the same location. $[17,18]$

From this image one can see that probes have been developed to integrate OCT optics into intravascular catheters to facilitate rotational and linear imaging capabilities. High speed data acquisition systems have enabled catheter based imaging to be preformed by manually or automatically feeding the probe through the vasculature during image acquisition. The OCT image in Figure 1.9 was made using a probe from Lightlab [19] (the current state of the art in radial OCT imaging). The OCT image was taken using a $1300 \mathrm{~nm}$ source with an $80 \mathrm{~nm}$ bandwidth. The image has a $1.25 \mathrm{~mm}$ maximum penetration depth with an axial resolution of about $13 \mu \mathrm{m}$ compared to the IVUS image with a $5 \mathrm{~mm}$ penetration depth and an axial resolution of about $98 \mu \mathrm{m}$ [18]. The superior penetration depth is what has kept IVUS imaging as the standard for intracoronary imaging, but the superior resolution of OCT has sparked quite some interest.

The visualization of vulnerable plaques in the coronary or carotid arteries and the evaluation of their potential for progression or rupture is an ideal application for cardiovascular OCT imaging. Ideally this evaluation would be performed after the area 
in question is localized by another imaging modality like CT, ultrasound, or fluoroscopy. Intravascular OCT is limited by tissue penetration depth and blood induced signal attenuation, although the signal attenuated by the blood can be reduced by a saline flush or temporary vascular balloon occlusion before imaging. [17, 19]

\subsection{Objective}

The objective of this thesis project is to develop a rotational catheter based OCT imaging system capable of three dimensional imaging in vivo via trans-lumenal methods. This objective was put forth in order to further the development of technology within the OCT group in Toronto while creating a system that is an improvement on current designs. The most significant improvement will be an increase in rotational speed. The highest published rotational rate to date is 108 rotations per second [20]. A faster imaging rate will improve the capability of the system to visualize rapid dynamic events (eg. moving leaflet of heart valves). The faster speed will also reduce the effects of motion artifacts due to physiological and/or patient motion. Furthermore, a faster frame acquisition rate will allow for a larger 3-D imaging volume while maintaining imaging resolution during a catheter pull-back aided by a limited time window of saline flush or a temporary vascular balloon occlusion for in vivo vascular imaging. The development of this probe will provide the medical imaging community with a wider range of possible studies that would include trans-lumenal vascular imaging, trans-esophageal imaging, and three dimensional monitoring for photodynamic therapy (PDT). The hypothesis for this thesis is that a rotational catheter based OCT imaging system capable of three dimensional imaging in vivo via trans-lumenal methods can be successfully developed. 


\section{CHAPTER 2: ROTARY SYSTEM DESIGN}

This chapter describes the evolution of the rotary system from the first CAD design to the current working prototype. The chapter will also detail the probe development and construction.

\subsection{Main System Design}

The first iteration of the rotary pullback system design is shown as an AutoCAD sketch in Figure 2.1.

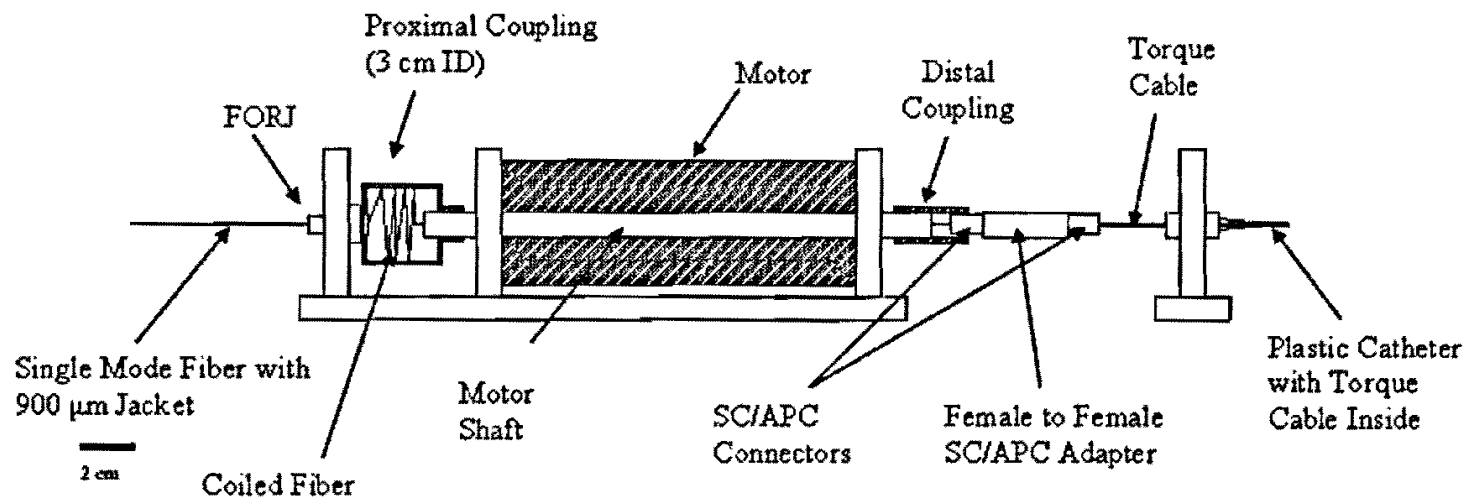

Figure 2.1 - CAD drawing of the first iteration of the rotary pullback system design

The laser light coming from the OCT source is lead to the rotary system by a single mode fiber that is connected to a fiber optic rotary joint (FORJ). A FORJ facilitates the rotation of an optical fiber. The fiber on the proximal end (stator) of the FORJ stays stationary as the fiber on the distal end (rotor) rotates. The fiber leaving the FORJ in Figure 2.1 goes through the proximal coupling which couples the FORJ to the shaft of the motor through which the fiber passes. The FORJ that was used is a MJX series joint from Princetel [21]. Figure 2.2 is a 3-D CAD drawing of the FORJ. 


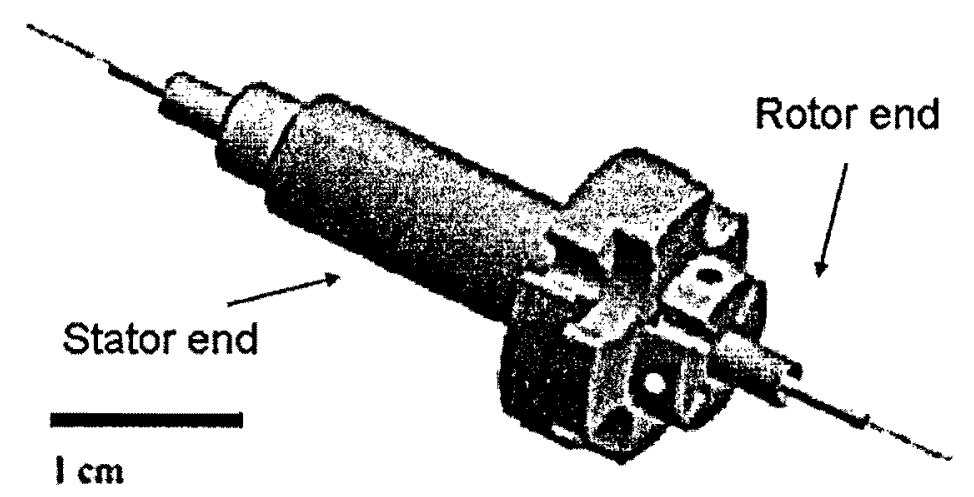

Figure 2.2 - 3-D CAD drawing of the fiber optic rotary joint (FORJ).

The power loss data through the FORJ was in question, so the power loss was measured manually. A $16 \mathrm{kHz}$ frequency swept laser from Thorlabs [22] was used to test the insertion loss. The power leaving the joint was measured with the rotor side of the joint positioned at 8 different angles. A position was chosen as $0^{\circ}$ and measurements were taken in $45^{\circ}$ increments from that point. The results were as follows:

\begin{tabular}{|l|c|}
\hline Tuning on $(\mathrm{mW})$ & 7.63 \\
\hline Tuning off $(\mathrm{mW})$ & 18.31 \\
\hline
\end{tabular}

Table 2.1 - Frequency swept laser power.

\begin{tabular}{|c|c|c|c|c|c|c|c|c|c|}
\hline Angle Rotated & $0^{\circ}$ & $45^{\circ}$ & $90^{\circ}$ & $135^{\circ}$ & $180^{\circ}$ & $225^{\circ}$ & $270^{\circ}$ & $315^{\circ}$ & Average \\
\hline Power Reading (mW) & 4.35 & 4.39 & 4.78 & 4.41 & 3.83 & 3.41 & 3.59 & 3.62 & 4.07 \\
\hline
\end{tabular}

Highest loss $=-3.5 \mathrm{~dB}$, Lowest Loss $=-2.03 \mathrm{~dB}$, Average loss $=-2.37 \mathrm{~dB}$

Table 2.2 - Power through FORJ with tuning on.

\begin{tabular}{|c|c|c|c|c|c|c|c|c|c|}
\hline Angle Rotated & $0^{\circ}$ & $45^{\circ}$ & $90^{\circ}$ & $135^{\circ}$ & $180^{\circ}$ & $225^{\circ}$ & $270^{\circ}$ & $315^{\circ}$ & Average \\
\hline $\begin{array}{c}\text { Power Reading } \\
(\mathbf{m W})\end{array}$ & 10.30 & 10.08 & 11.02 & 10.30 & 8.69 & 7.30 & 7.58 & 7.67 & $\mathbf{9 . 1 2}$ \\
\hline
\end{tabular}

Highest loss $=-3.99 \mathrm{~dB}$, Lowest Loss $=-2.21 \mathrm{~dB}$, Average loss $=-3.03 \mathrm{~dB}$

Table 2.3 - Power through FORJ with tuning off. 


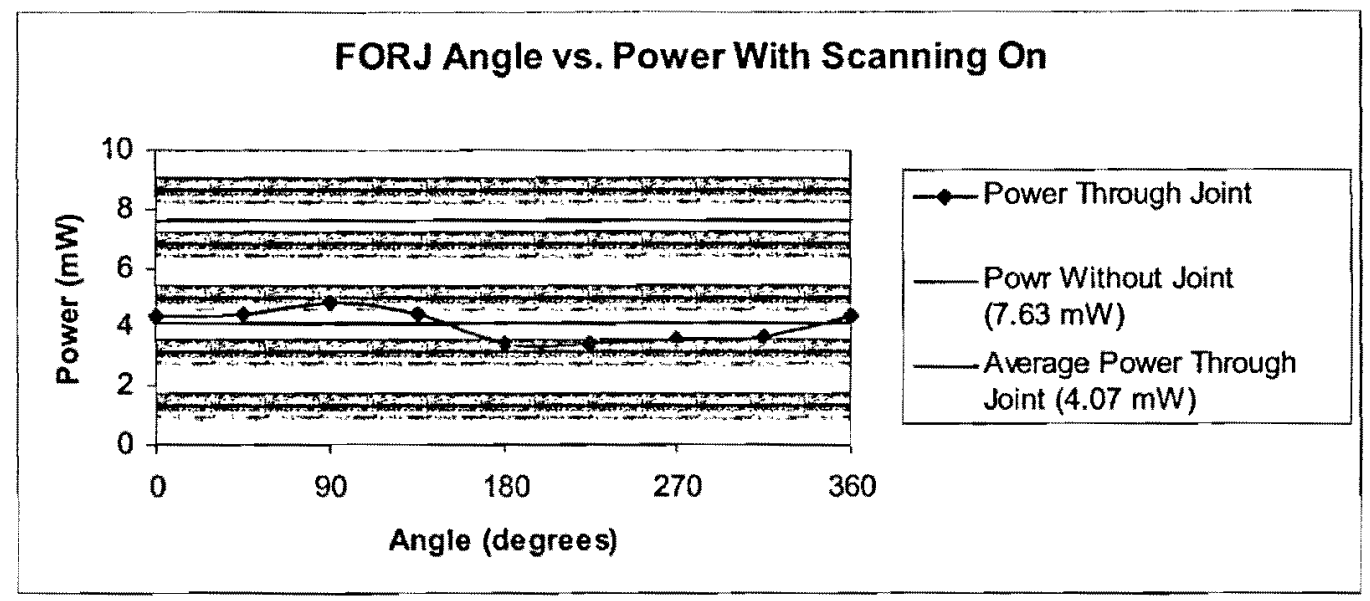

Figure 2.3 - Data from Table 2.2 in graphical form.

From Table 2.2 and Figure 2.3, one can see that average one way loss through the FORJ while the swept source laser is scanning is $-2.37 \mathrm{~dB}$. While imaging a sample, the light would have to pass through the FORJ then to the sample and back through the FORJ. That would mean that roughly $42 \%$ of the light leaving the system would not reach the sample and of the light reflected off the tissue only $42 \%$ would have the potential of reaching detector. This means that any losses attributed to the other components of the system must be kept to a minimum. The loss due to the FORJ is most likely due to a misalignment between the fiber in the stator and the fiber in the rotor. More information on the possible lateral, angular and longitudinal misalignments can be found in [23].

The motor that the FORJ is coupled to is a custom Neo70 motor from Parker Motion [24]. Figure 2.4 is a 3-D CAD drawing of the motor that was used. 


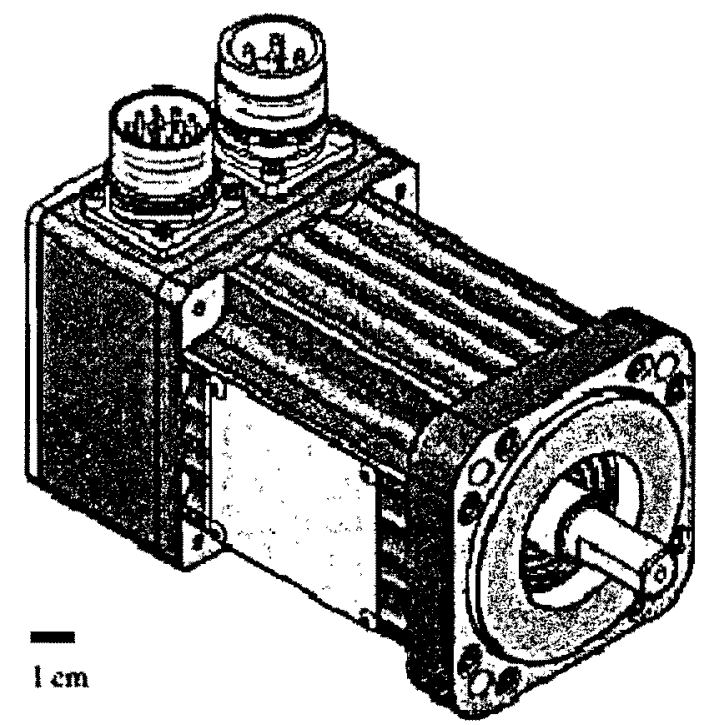

Figure 2.4 - 3-D CAD drawing of the rotary motor showing an isometric view

The motor was designed with a dual shaft and $5 \mathrm{~mm}$ through bore to pass the optical fiber through. Passing the fiber through the motor shaft allows the fiber to be directly driven. This eliminates any loss in rotational speed and radial stability associated with indirectly driving the fiber. The encoder has a pulse rate of 40000 pulses per second regardless of speed. Therefore, the motor gives an accurate angle reading with regards to the degree to which the motor has rotated. The motor was designed to reach a rotation rate of $200 \mathrm{~Hz}$. This speed will allow for image acquisition rates up to 200 frames per second. This is significantly higher than the highest published acquisition rate to date of 108 frames per second [20]. As mentioned earlier, the faster imaging rate will improve the capability of the system to visualize rapid dynamic events. The faster speed will also reduce the effects of motion artifacts due to physiological and/or patient motion. Furthermore, a faster frame acquisition rate will allow for a larger 3-D imaging volume while maintaining imaging resolution during a catheter pull-back aided by a limited time 
window of saline flush for in vivo vascular imaging. A saline flush is required to create a brief moment when highly scattering blood is displaced.

The distal coupling in Figure 2.1 connects the connector end of the radial probe to the motor. A SC-APC connector was chosen for the motor end because it allows the mating sleeve to be easily clipped on and off for different radial probes with different connectors. The commonly used FC-APC connectors are not as versatile because they may tighten or loosen as the motor rotates due to their screw like threading. SC-APC connectors were chosen for the radial probes for the same reasons. Figure 2.5 shows examples of FC-APC and SC-APC connectors.
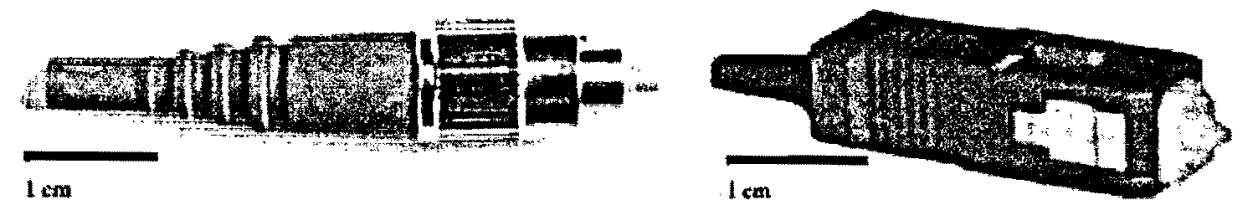

Figure 2.5 - Left: Example of a FC-APC connector. Right: Example of a SC-APC connector

The support on the right side of Figure 2.1 holds the outer catheter of the radial probes in place to allow for the translational motion of the optics during a scan. Figure 2.6 shows the support holding an outer catheter. More information about the catheters can be found in the next section. 


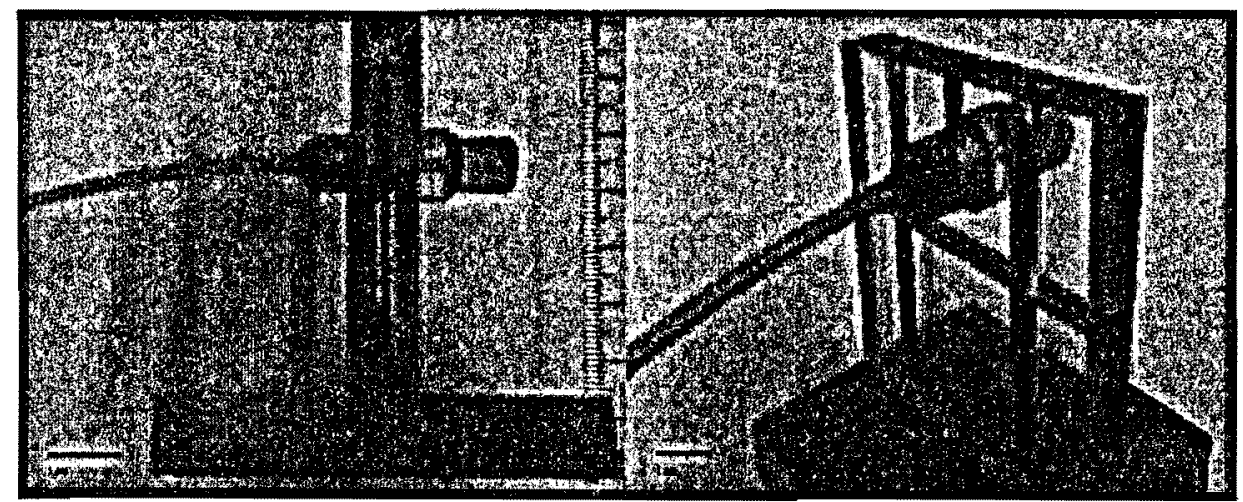

Figure 2.6 - Side and isometric views of the catheter support holding an outer catheter. The scale bars are $1 \mathrm{~cm}$.

The system is mounted on a linear motion stage to facilitate the translational motion. For the initial prototypes a simple 436 series linear stage from Newport [25] was used. The stage is driven by a Zaber [26] T-LA60A miniature linear actuator. This combination allows for a linear range of $6 \mathrm{~cm}$ at a max speed of $4 \mathrm{~mm} / \mathrm{s}$.

It is worthy to note that the proximal coupling in Figure 2.1 has coiled fiber inside. The coiled fiber acts as extra fiber that allows for more than one attempt at splicing the connector to the fiber at the distal end of the motor. If the first attempt at splicing the connector fails then some of the fiber can be uncoiled to make another attempt. On the first prototype the coiled fiber was moved to the distal coupling and the rotor end of the FORJ was inserted directly. into the motor shaft. Figure 2.7 shows images of a prototype for the aforementioned distal coupling design. The figure on the right shows the coiled fiber exposed. 


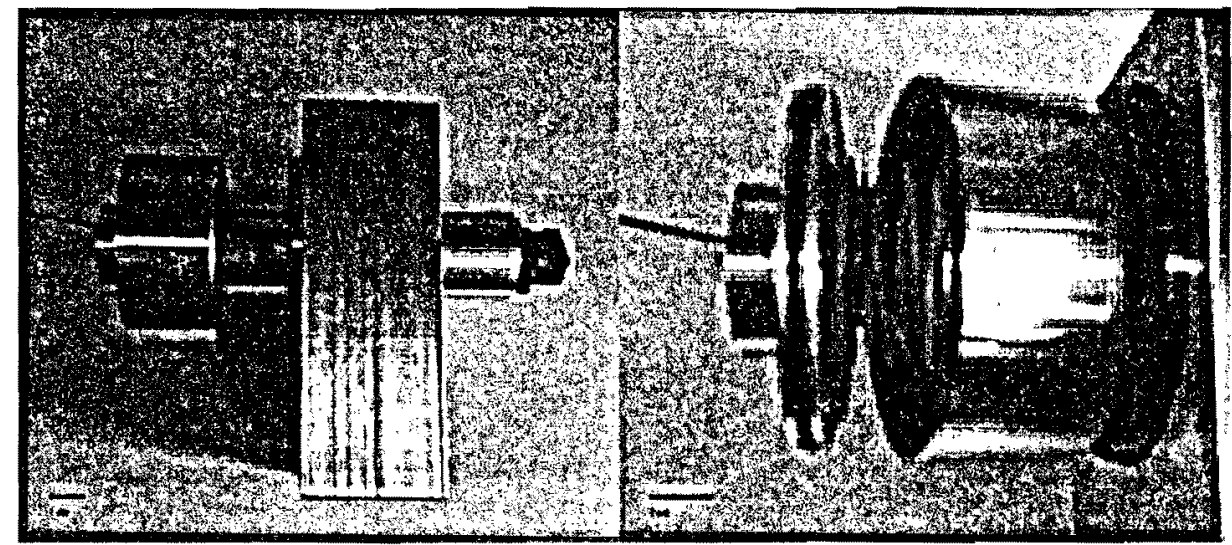

Figure 2.7- Distal coupling of the first prototype of the rotary pullback system. The scale bars are $1 \mathrm{~cm}$.

The chamber for this distal coupling has an inner diameter of $5 \mathrm{~cm}$. This is $2 \mathrm{~cm}$ greater than the minimum recommended diameter for single mode fiber. Putting the coiled fiber chamber on the distal end added significant length to the coupling so a support with a rotational bearing was added. Figure 2.8 shows the first prototype of the rotary pullback system with a probe from industry attached.

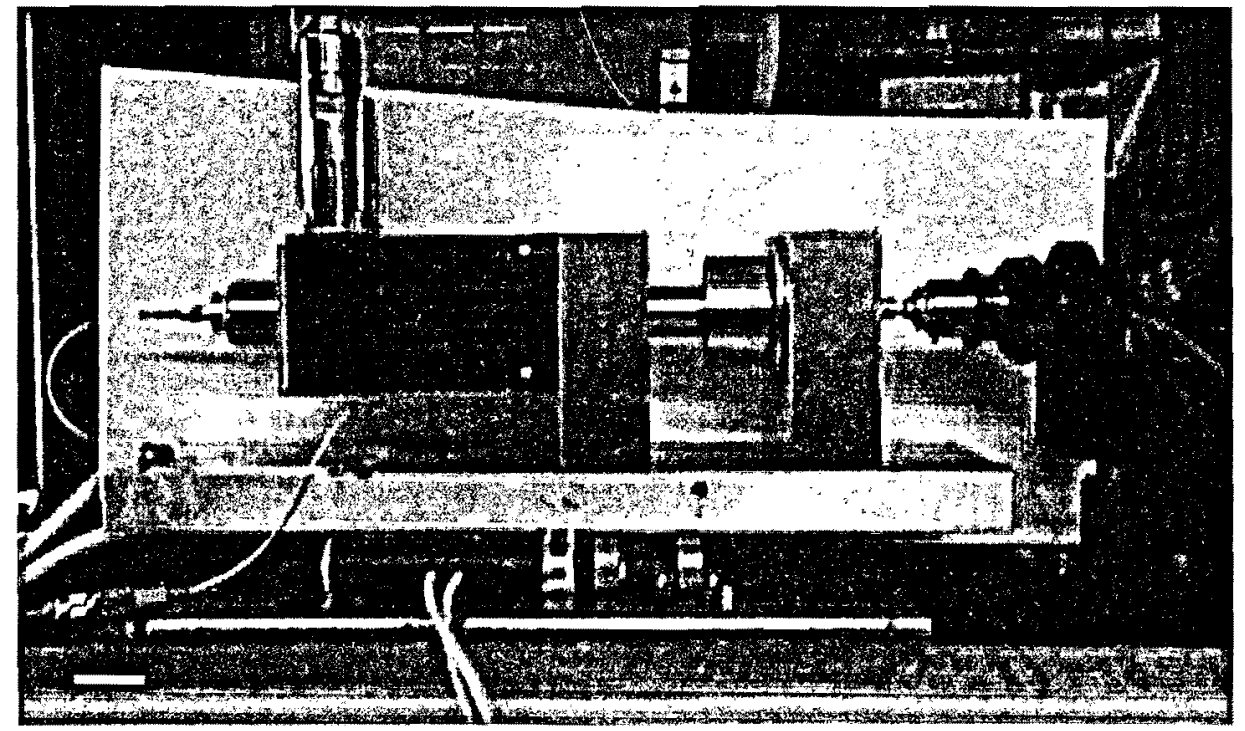

Figure 2.8 - First prototype of the rotary pullback system. 
An image was taken of a human index finger and thumb grasping the probe. The resulting image can be seen in Figure 2.9. The image was taken at a time before a program was made to create the radial representations of images that most are used to seeing with radial probes. The index finger is on the right (top if radial) and the thumb is on the left (bottom if radial). Part of the thumb is missing because the A-scan acquisition rate was not matching the radial speed.

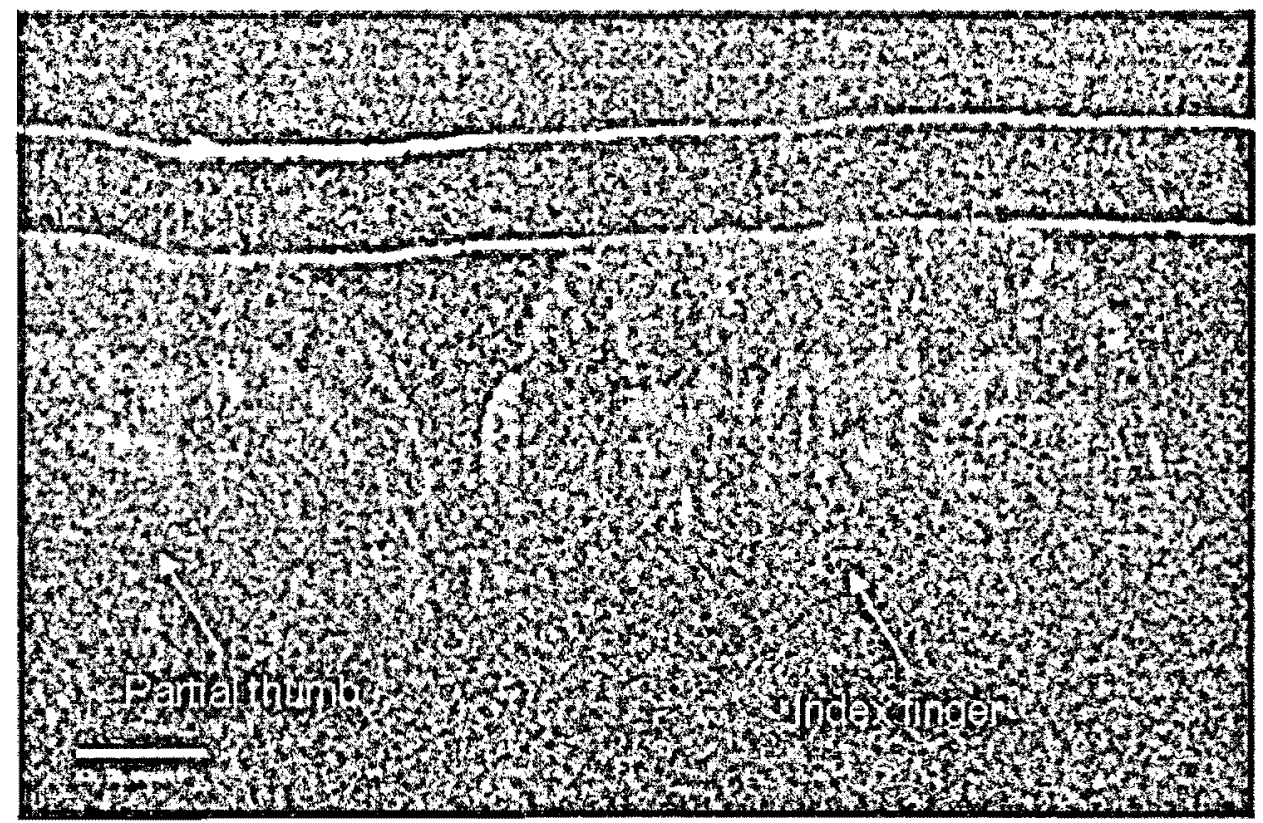

Figure 2.9 - Image of a thumb and index finger grasping a radial probe from industry.

From Figure 2.9 it can be clearly seen that the signal off the fingers was just barely stronger than the noise. This was an indication that the system was causing a large loss of power. The cause of the power loss was narrowed down to the coiled fiber inside the distal coupling. The optimal orientation of the fiber (ie. a bending radius more than 3 $\mathrm{cm}$ ) was not being maintained while the probe was spinning. This resulted in a total average one way system loss of about $6 \mathrm{~dB}$. This meant that $75 \%$ of the light from the 
laser was not reaching the sample and at least $75 \%$ of the signal from the tissue was lost before it reached the detector. For this reason the distal coupling had to be redesigned. To eliminate any loss due to fiber orientation the new coupling had to keep the fiber straight at all times. In order to do this the distal coupling had to be machined after the connector was spliced to the fiber at the distal end of the motor. This was done so that the coupling would be the correct length to fit perfectly over the entire fiber on the distal end. Figure 2.10 is a CAD drawing of the distal coupling used for the current prototype.

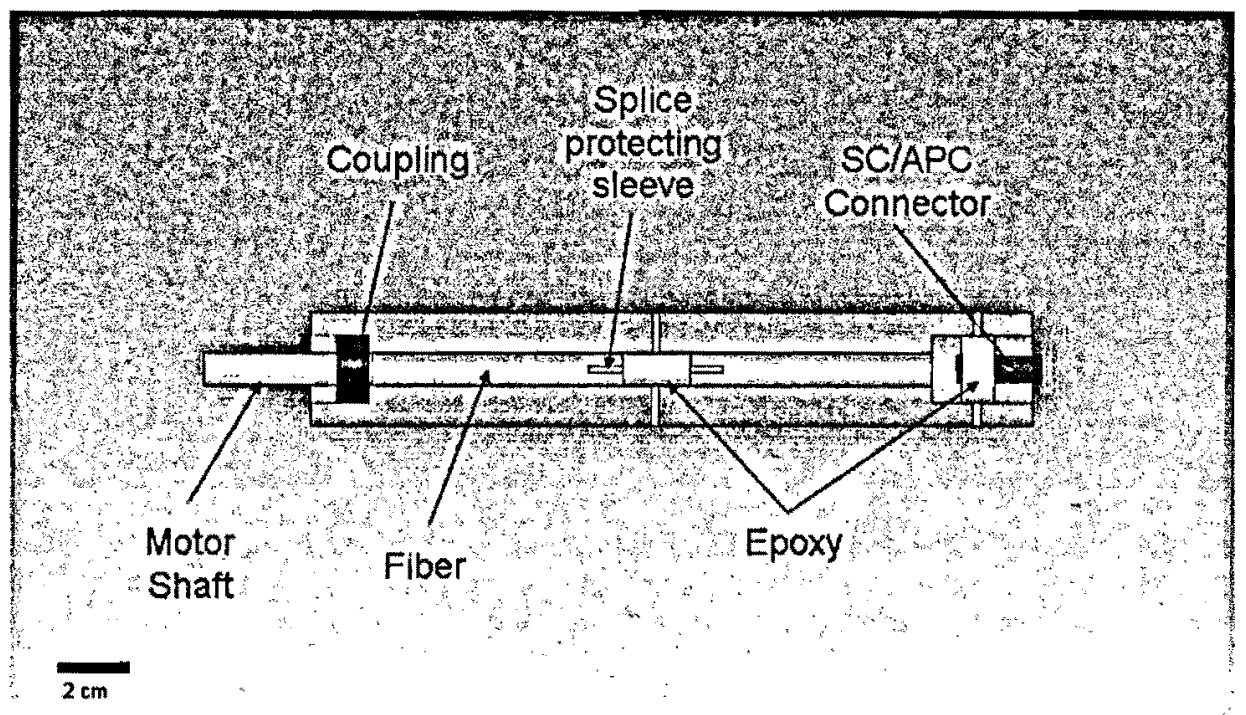

Figure 2.10 - Distal coupling of the current rotary pullback system prototype.

The black coupling in Figure 2.10 was put on the motor shaft before the connector was spliced on. The connector was pushed through the distal coupling. The hollow center of the distal coupling was filled entirely with epoxy in order to hold the fiber, splice protection sleeve, and connector in place while the coupling spins. The epoxy is shown only over the connector and splice protection sleeve in order to reveal the positioning of everything within the distal coupling. Figure 2.11 shows the current prototype. 


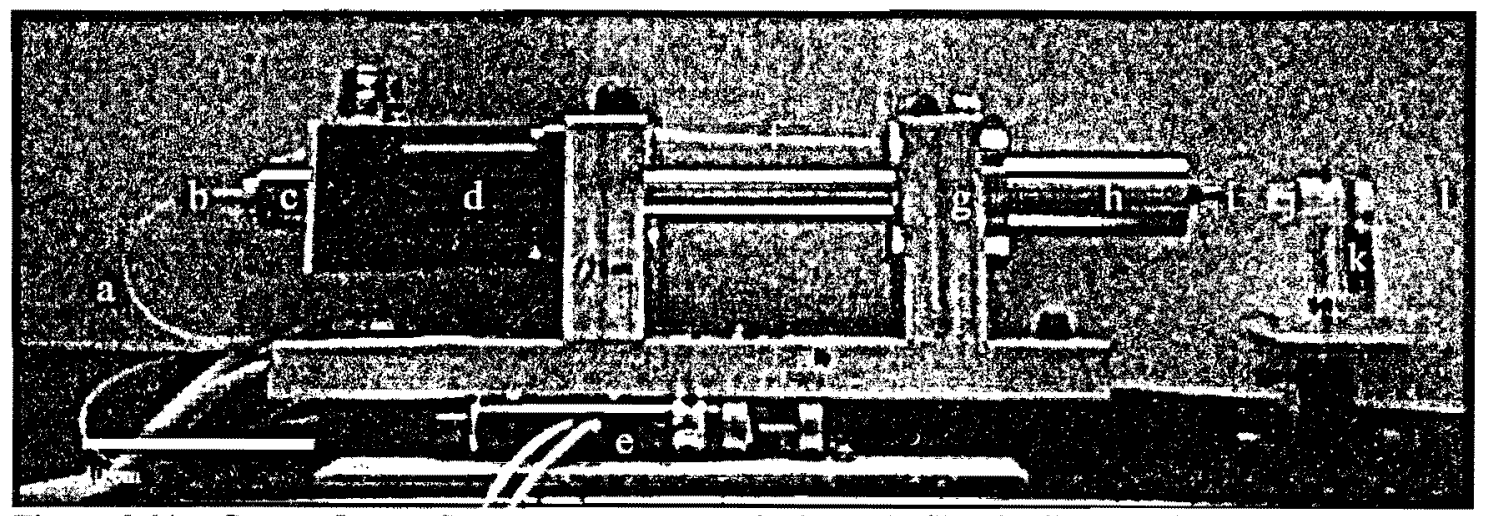

Figure 2.11 - Current Rotary System Prototype. a) Single mode fiber leading to a laser source, b) Stator end of the fiber optic rotary joint (FORJ). c) Chamber covering the rotary end of the FORJ, which is coupled to the hollow motor shaft. The chamber protects the user in the event that the FORJ malfunctions and breaks apart at high speeds. d) Custom Neo70 motor from Parker Motion. e) Zaber T-LA60A miniature linear actuator, f) Shaft coupling the motor, rotating FORJ fiber, and SC/APC mating sleeve. g) Post with a high speed bearing used to carry the coupling load. h) Protective covering over the mating sleeve. i) SC/APC connector of a probe plugged into the system. j) Adapter used to hold the outer catheter of the probes in place during a linear pull-back. The adapter also has a threaded end to facilitate the addition of a cap to seal the probe for sterilization. k) Post used to hold the probes in place. 1) Probe leading to a sample to be imaged.

The loss for the current system $(\sim-2.50 \mathrm{~dB}$ on average $)$ is roughly equivalent to the loss attributed to the FORJ on its own. Figure 2.12 shows some images taken with same rotary probe from industry as earlier. The signal to noise ratio for these images is clearly much better than in the image taken with the first prototype (Figure 2.9). The image on the left is of a thumb (bottom) and index finger (top) grasping the catheter as earlier. The image on the right is of the probe taped to an IR card. The details on how the images were reconstructed will be covered in Chapter 3. 

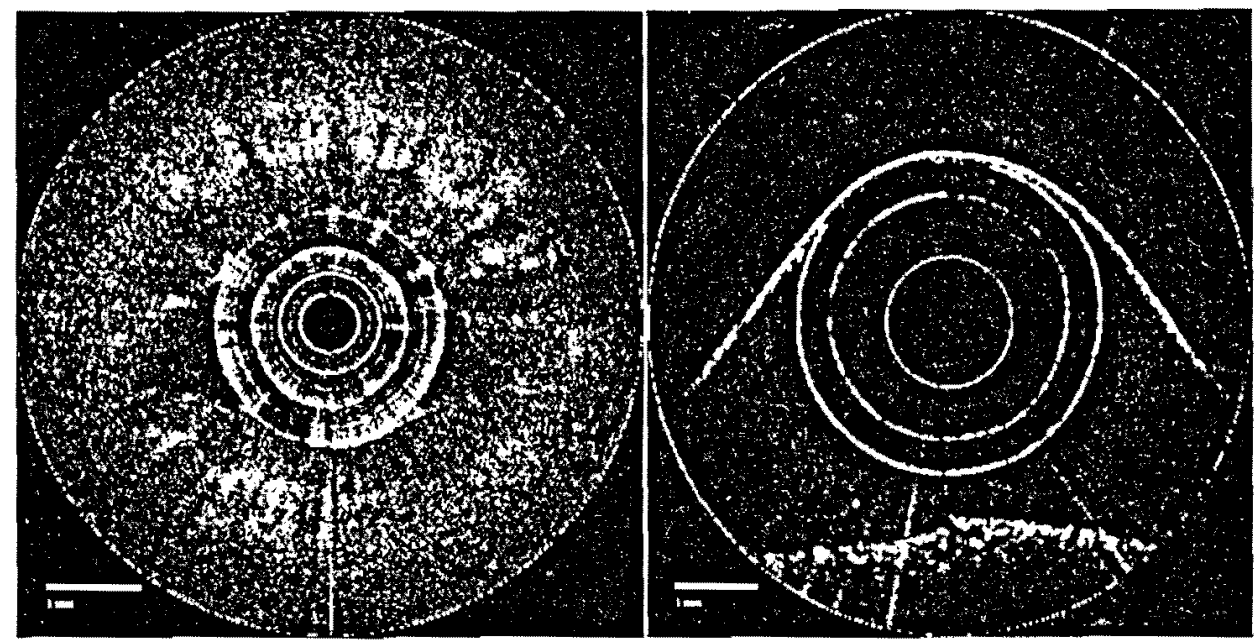

Figure 2.12 - Left: 2-D image of a thumb (bottom of image) and index finger (top of image) grasping a catheter. Right: 2-D image of a probe taped to an IR card. The scale bar is $1 \mathrm{~mm}$.

\subsection{Probe Design}

\subsubsection{Catheters}

The probes built for the system were modeled after the Intravascular Ultrasound (IVUS) [27] miniature torque cable design. The torque cable is a tightly wound stainless steel cable with an outer diameter of $0.7 \mathrm{~mm}$ that acts as a catheter that rotates with the optical fiber. The torque cable has a unique quality that allows the degree of rotation at the proximal end of the probe to be almost exactly the same as the degree of rotation at the distal end of the probe. This eliminates any unwanted twisting in the fiber. The torque cables used for the probes made during this project were taken directly from expired IVUS catheters. In order to utilize a torque cable from an IVUS probe, the imaging end and the proximal end of the cable are cut with very sharp pinching pliers in order to detach the cable from the IVUS system as cleanly as possible. The ultrasound fiber is then removed and replaced with a single mode optical fiber. Figure 2.13 shows 
the imaging end of an IVUS probe. The rotating drive shaft in the image is the torque cable.

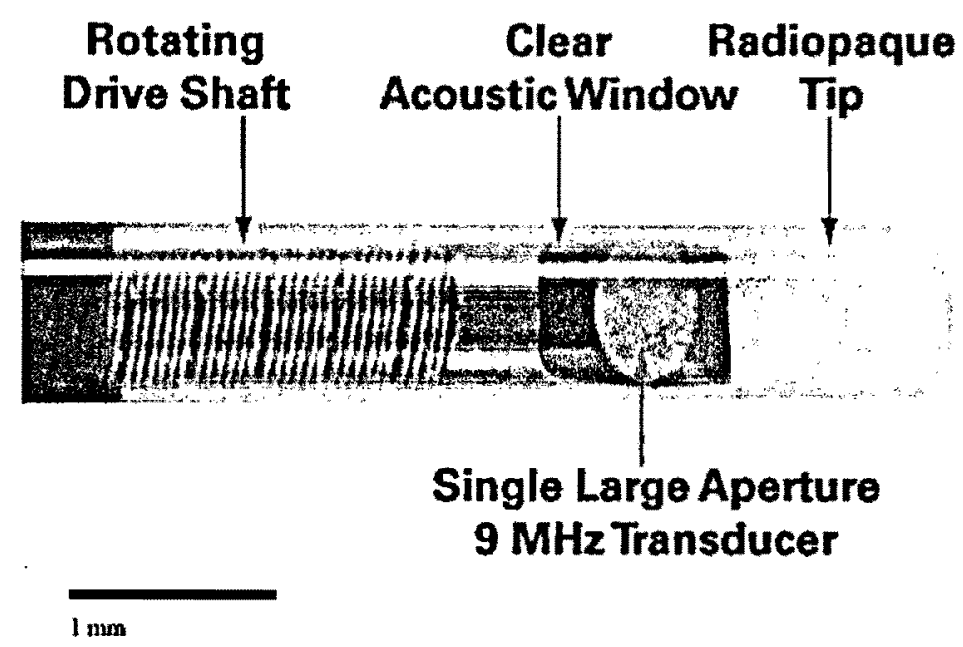

Figure 2.13 - Imaging end of an Intravascular Ultrasound probe [27].

The outermost catheter is a biocompatible USP Class VI Teflon tube [28] with an outer diameter just under $1 \mathrm{~mm}$. The outer catheter is attached to the adaptor listed as " $\mathrm{j}$ " in Figure 2.11 with epoxy. The adapter has a threaded end so that a cap can be screwed onto the catheter. The cap prevents the outer catheter from allowing moisture in during the cleaning process. Figure 2.14 shows the adaptor with and without the cap. Figure 2.15 shows a completed probe ready for sterilization. A prototype of this probe design has been put through a cleaning process in a sterilizing machine used to clean reusable endoscopic devices at St. Michaels Hospital in Toronto. The design was proven effective. 


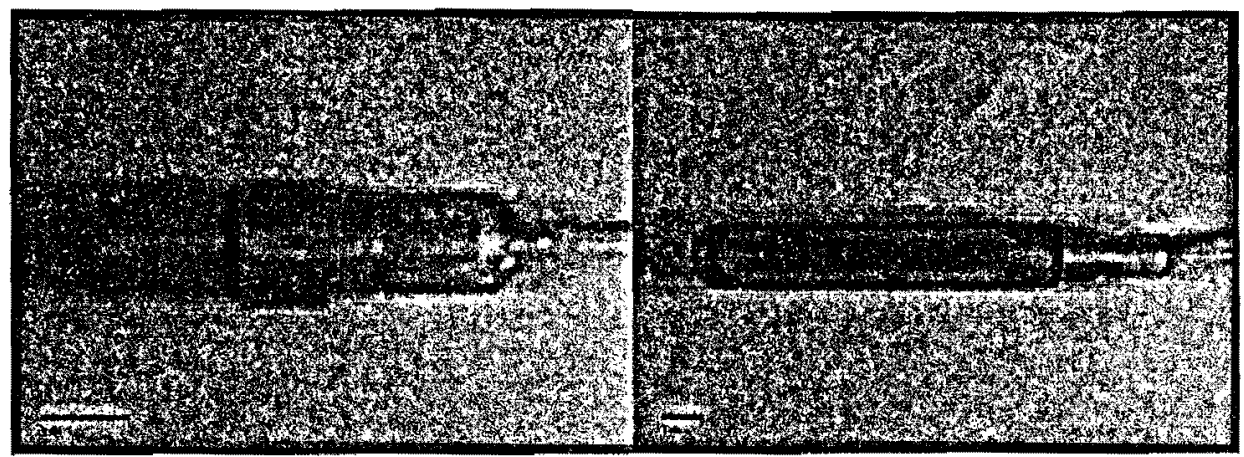

Figure 2.14 - Left: Adaptor end of an outer catheter. Right: Adaptor with cap. The scale bars are $1 \mathrm{~cm}$.

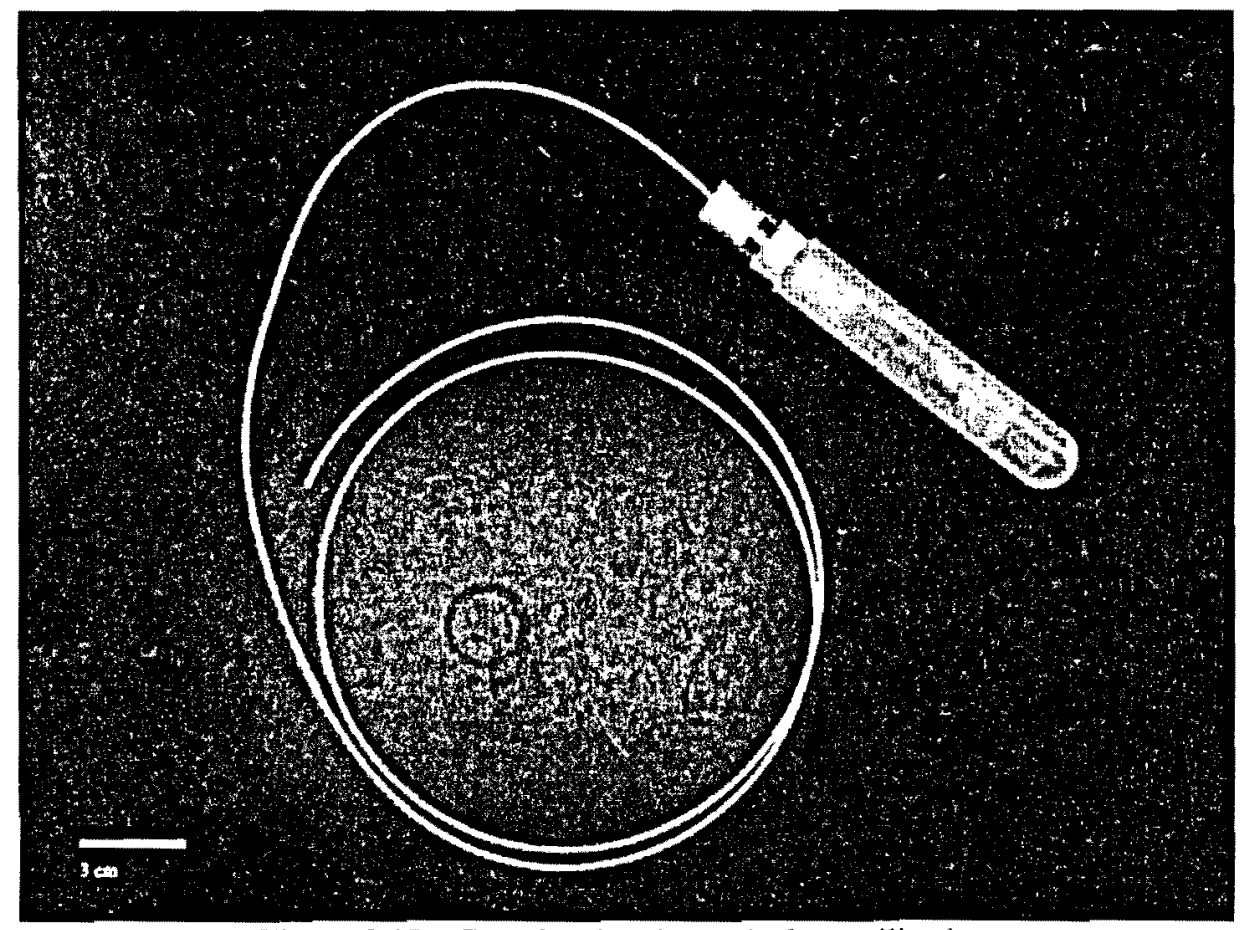

Figure 2.15 - Completed probe ready for sterilization. 


\subsubsection{Optics}

The fiber portion of a probe is comprised of single mode (SM) fiber with an arrangement of graded-index (GRIN) and coreless (CL) fibers at the tip of the distal end to form the optics. The GRIN and CL fiber are cut at certain lengths to achieve certain working distances (focal distance from the lens surface), depths of field (two times the Raleigh range), and spot sizes (beam waist diameter). SM fibers are optical fibers which are designed to support only a single mode of light. The core of a common single mode fiber is around $9 \mu \mathrm{m}$. The core of SM fiber is surrounded by cladding which puts the outer diameter of the fiber to $125 \mu \mathrm{m}$. GRIN fibers have a larger core at about $100 \mu \mathrm{m}$ with the cladding layer putting the outer diameter to $140 \mu \mathrm{m}$. The index of refraction is highest at the center and decreases with radial distance from the center axis in GRIN fiber. This property is used to expand the beam from the SM fiber and then focus it at some point in front of the GRIN fiber in an OCT probe. The following equation closely describes the refractive index of a GRIN fiber lens [29]:

$$
n(r)=n_{o}\left(1-\frac{g^{2}}{2} r^{2}\right)
$$

where, $r$ is the radial position from the axis, $n_{0}$ is the refractive index at the center axis, and $\mathrm{g}$ is the gradient constant given by the manufacturer. $\mathrm{CL}$ fibers are fibers without a wave guiding structure. The CL fiber is a uniform material with roughly the same index of refraction as the core of a SM fiber and a diameter of $125 \mu \mathrm{m}$. For side viewing probes the CL fiber is the last stage of the optics and is polished to an angle of 50 degrees. The polished surface of a CL fiber is a totally reflective surface that directs the focused light to the side of the probe. An angle of 50 degrees is chosen instead of 45 degrees to avoid any saturation issues involved with the light being reflected directly 
back at the fiber. The optical ends of the probes used for the work presented in this thesis were polished manually using a 50 degree angled polishing block. The probes were placed on the block and held in place with polishing glue (Phenyl Salicylate). The polishing glue was then polished with $9 \mu \mathrm{m}$ polishing paper to flatten its surface. The flat glue surface was then polished with $1 \mu \mathrm{m}$ polishing paper until the tip of the probe started to appear. The probe tip was then polished with $0.3 \mu \mathrm{m}$ polishing paper until the probe tip was completely angle polished to 50 degrees. Figure 2.16 shows a polished probe tip in the midst of polishing glue.

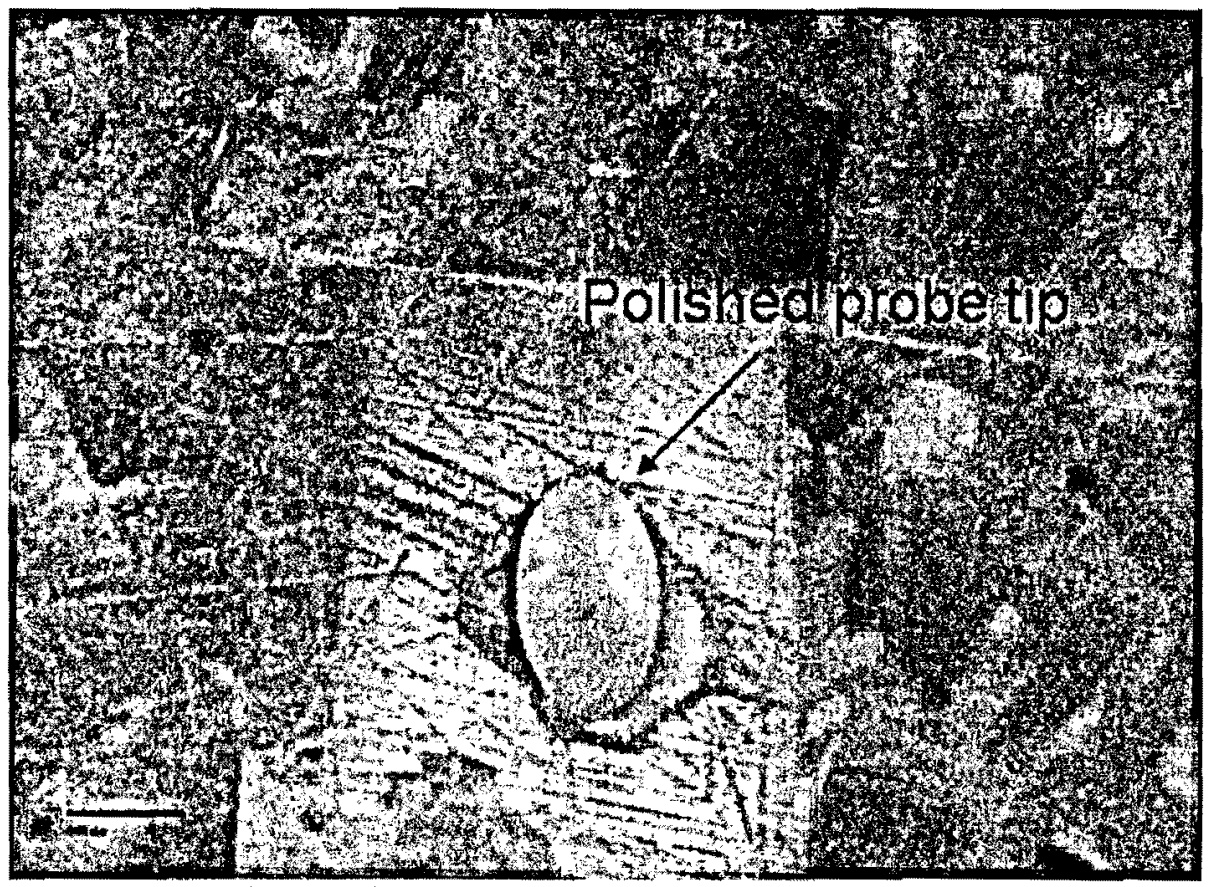

Figure 2.16 - Polished probe tip in the midst of polishing glue. The scale bar is $100 \mu \mathrm{m}$.

A typical side viewing OCT probe has a $0.45 \mathrm{~mm}$ GRIN fiber followed by an angle polished coreless fiber for its optics. The first radial probes were built with this arrangement. Figure 2.17 is a radial image taken with one of those probes. The image is of a probe taped to an IR card. 


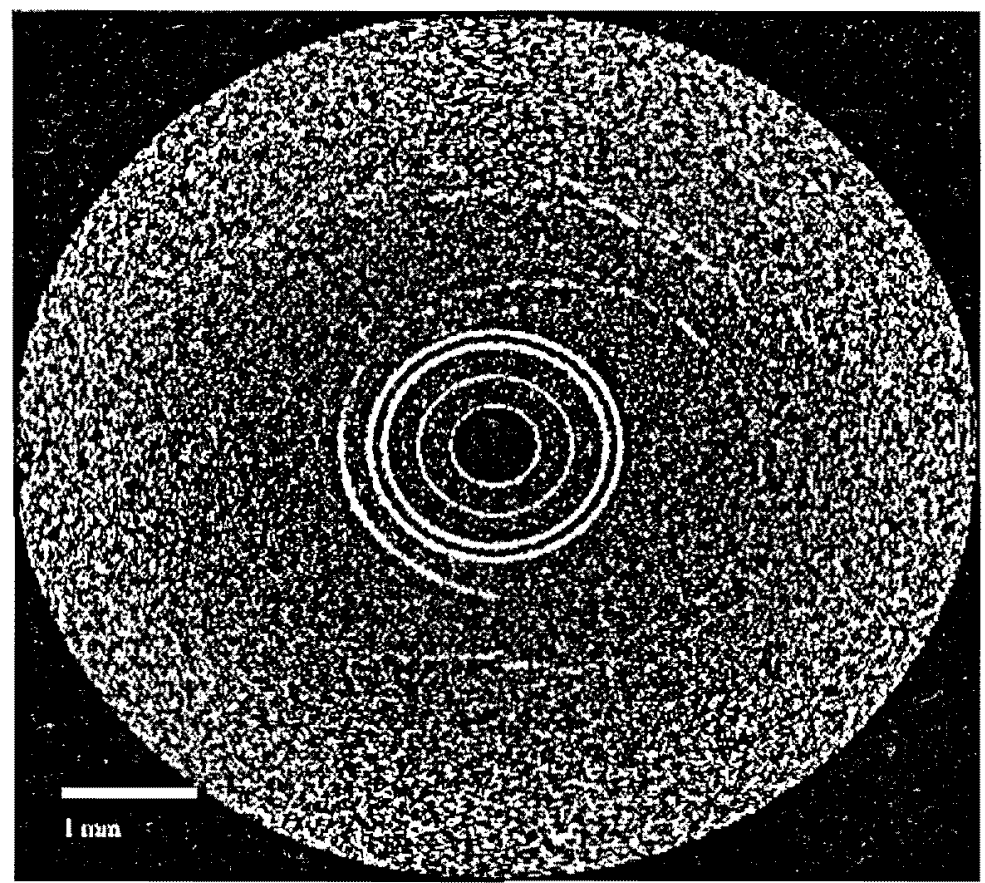

Figure 2.17 - Radial image of a probe with $0.45 \mathrm{~mm}$ GRIN optics taped to an IR card.

The signal from the IR card was barely above the noise. There are a couple reasons why this may have occurred. The main reason is that a probe manufactured with a $0.45 \mathrm{~mm}$ GRIN section has a short working distance of about $0.38 \mathrm{~mm}$. This design flaw leaves the focal point of the probe well within the outer catheter. Another reason is that the polished surface of the optics may not have been completely smooth. A newly polished probe tip still in the polishing glue, as in Figure 2.16, was analyzed for its smoothness before a new arrangement for the optics was implemented. The polished surface was analyzed with a Wyko optical profiler [30] at the Institute for Optical Sciences at the University of Toronto and a scanning electron microscope (SEM) at the Mechanical Engineering Department at Ryerson University. Figures 2.18 and 2.19 are samples of the results from the optical profiler tests. 


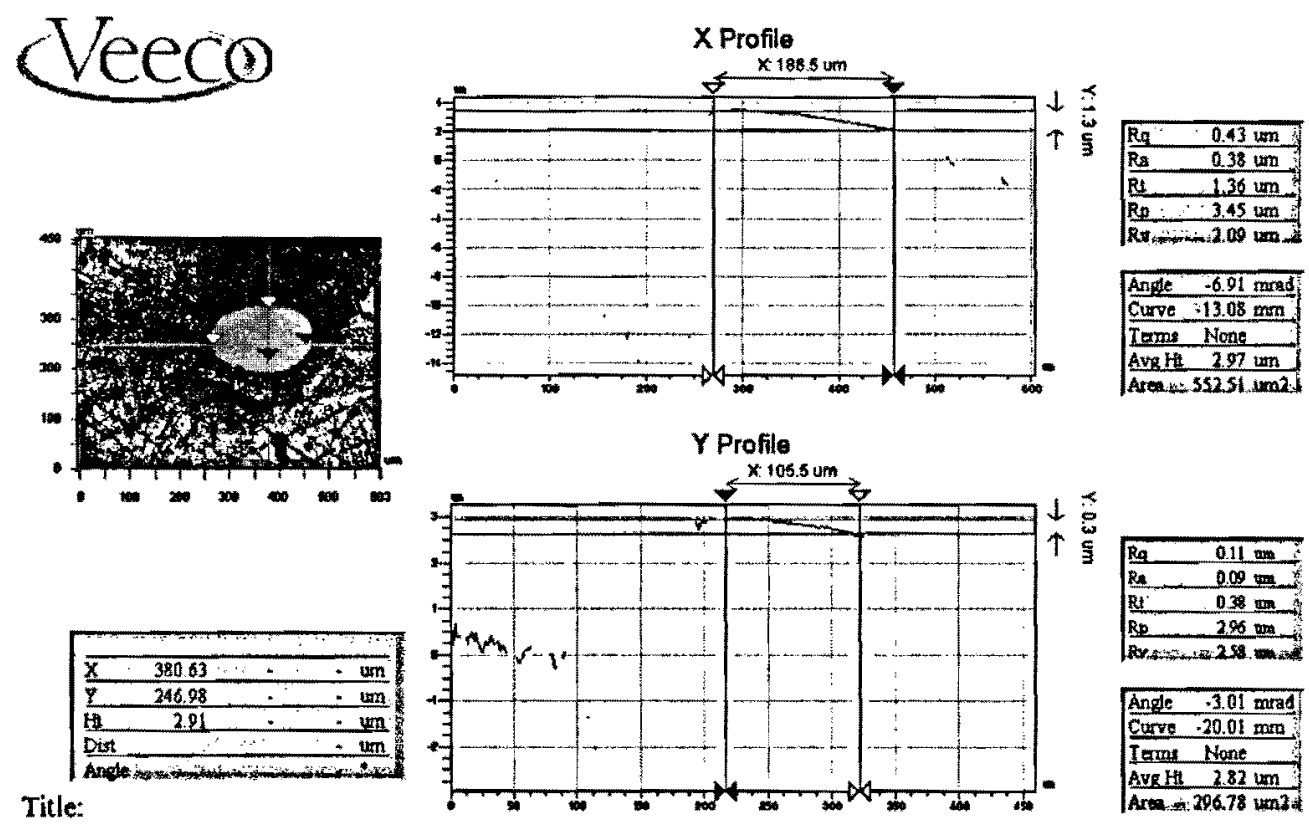

Note:

Figure 2.18 - Optical profiler results for the polished probe surface at an effective magnification of about $10 \mathrm{x}$. The top left image shows the probe tip in polishing glue. The red and blue lines on the probe tip correspond to the locations of the depth profiles shown with the red and blue plots in the graphs.
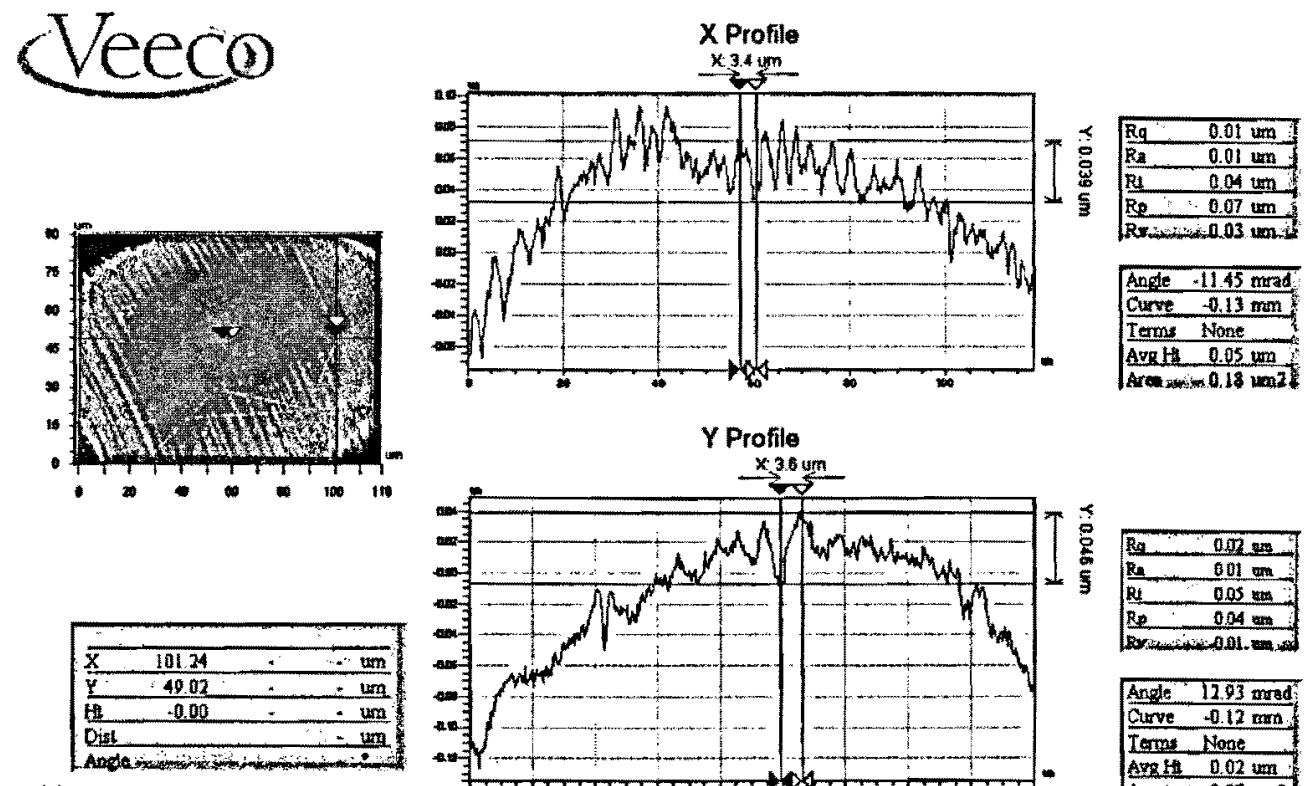

Title:

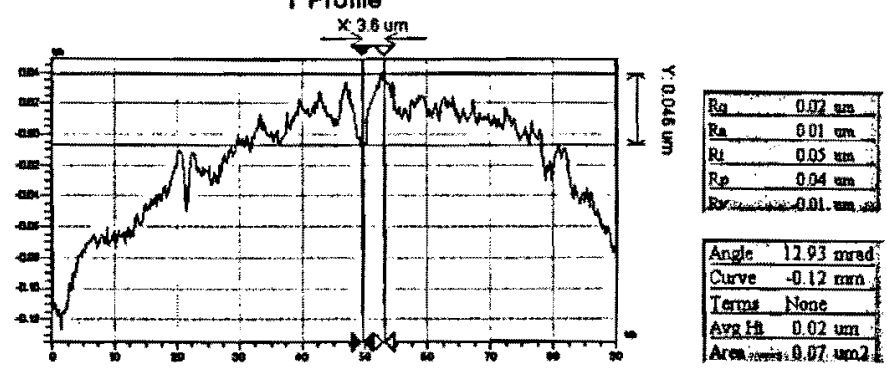

Note:

Figure 2.19 - Optical profiler results for the polished probe surface at an effective magnification of about 50x. The top left image shows the probe tip in polishing glue. The red and blue lines on the probe tip correspond to the locations of the depth profiles shown with the red and blue plots in the graphs. 
As can be seen in the figures, the polished surface of the probe was fairly smooth. The maximum possible surface roughness value for the polished probe tip came to be $\lambda / 34$ for a $1310 \mathrm{~nm}$ light source. Considering that the irregularities in the polished surface were significantly smaller than the wavelengths of light used for imaging, it is safe to say that the polished surface is not a cause for concern. The results from the SEM scan lead to a similar conclusion. Figure 2.20 shows some results from the SEM scan.
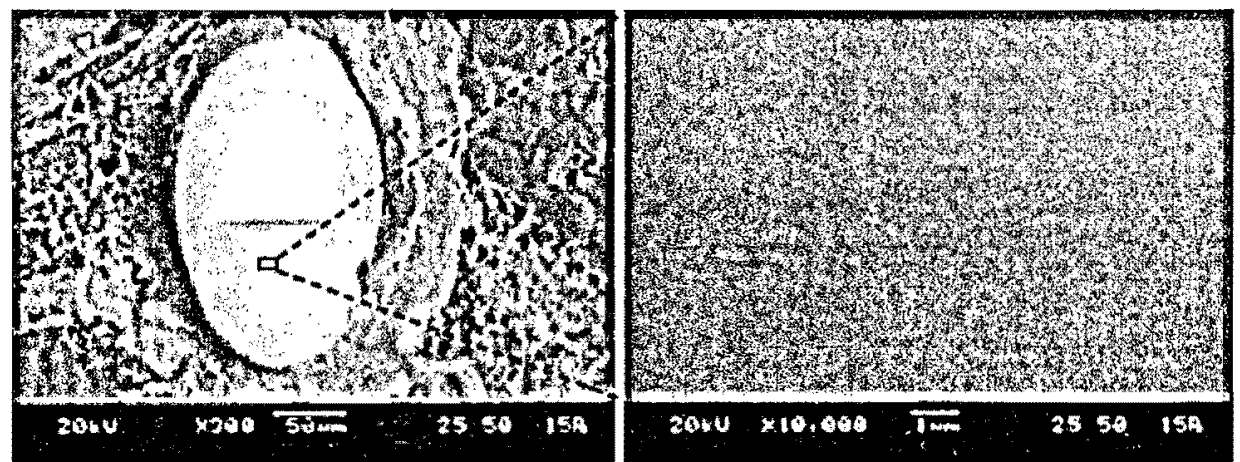

Figure 2.20 - SEM results of polished probe surface at a magnification of 300x (left) and a section of the polished surface at $10000 \times$ (right).

The images from the SEM imaging did not show any irregularities in the polished surface. Even at a magnification of $10000 x$ the surface appeared completely smooth. It is worthy to note that the SEM imaging revealed that the probe was polished right through the CL fiber. This is evident on the left of Figure 2.20. The top part of the polished surface is coreless fiber and the bottom part is GRIN fiber.

With the surface of the probes appearing not to be a factor, it was time to create a probe with a larger working distance. To accomplish this, the optics were constructed with a CL spacer before the GRIN section. The optics were constructed according to [29]. When the light enters the CL fiber from the SM fiber it begins to diverge at an angle of about 8 degrees off the central axis. This widens the beam before it enters the 
GRIN section and as a result the beam is wider when it hits the angled CL fiber, thus, creating a longer working distance. The sketch in Figure 2.21 shows the beam path through optics with a CL spacer.

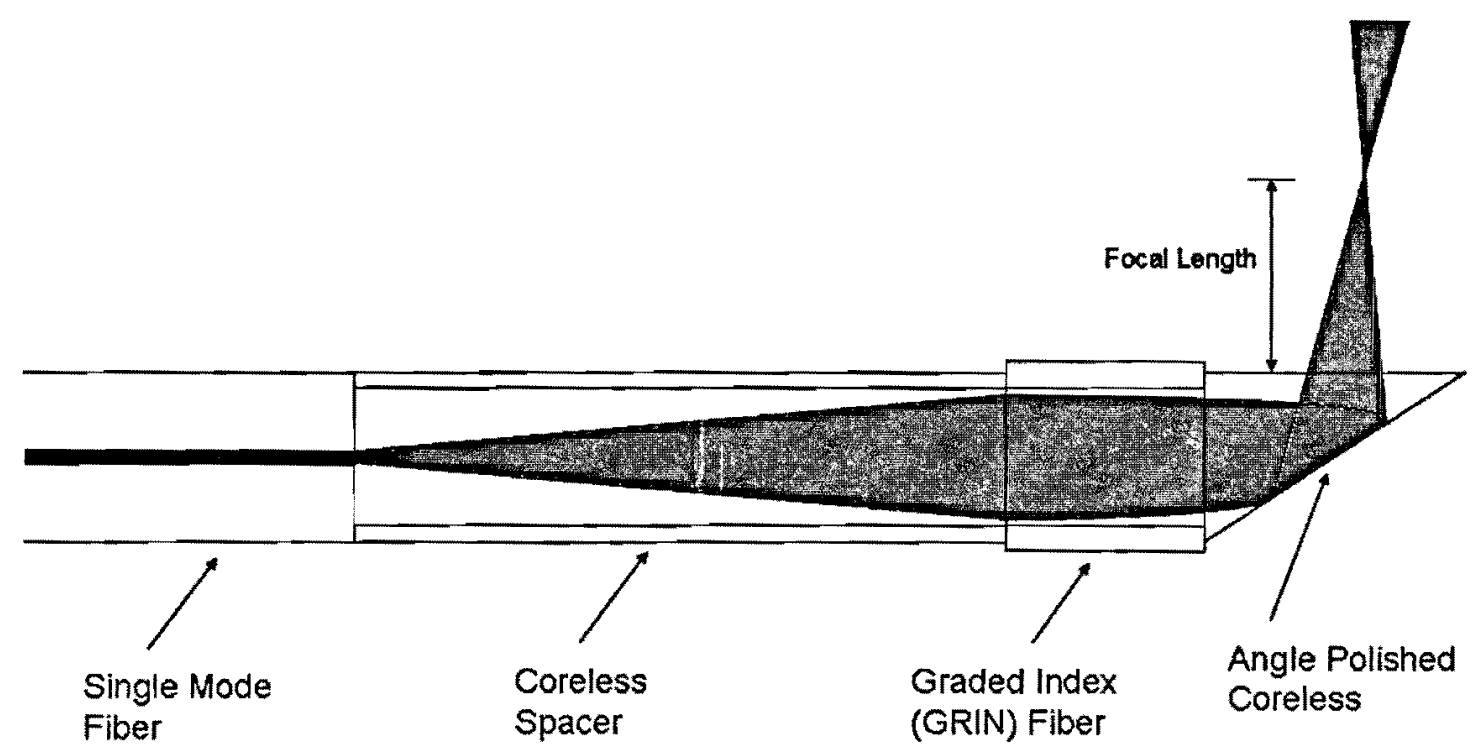

Figure 2.21 - Sketch showing the light path through the optics of a probe with a coreless fiber spacer.

A probe with the coreless fiber arrangement was characterized with a $1310 \mathrm{~nm}$ laser at 3 $\mathrm{mW}$ after the GRIN fiber stage was spliced on to the CL spacer (the forward looking stage) in order to check if the arrangement would be sufficient for a side viewing probe. A probe with a $0.626 \mathrm{~mm} \mathrm{CL}$ spacer and a $0.145 \mathrm{~mm}$ GRIN section was placed at various distances from a mirror and the power reflected back was measured. The resulting Gaussian plot can be seen in Figure 2.22. The distance with the highest power corresponds to the focal distance. The focal distance was $0.8 \mathrm{~mm}$. The full width at half maximum (FWHM) of the plot corresponds to the depth of focus (b). The depth of focus was found to be $0.6222 \mathrm{~mm}$. From equation (3), it is found that the spot size (lateral resolution) for this probe was $22.8 \mu \mathrm{m}$. This analysis shows that the CL spacer 
configuration will result in a longer working distance while maintaining a reasonable spot size.

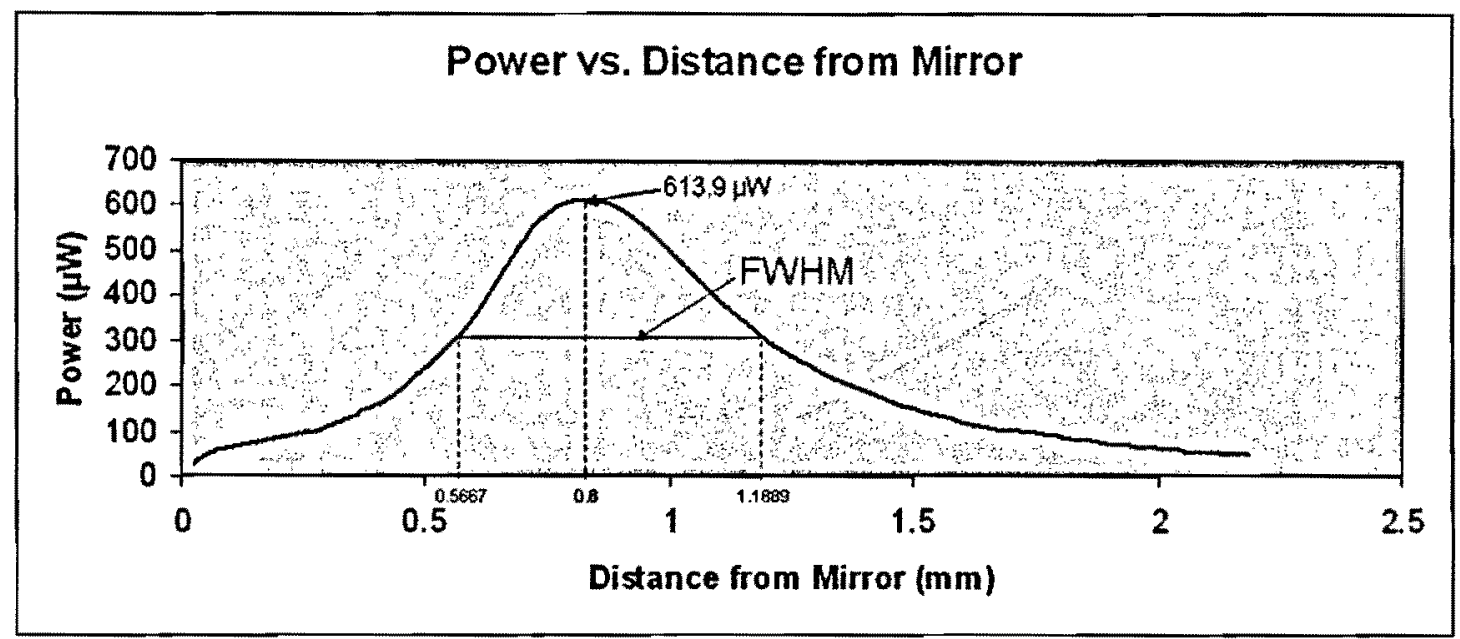

Figure 2.22 - Power profile for coreless spacer probe characterization. A 1310nm laser with a power of 3 $\mathrm{mW}$ was put through a probe at various distances from a mirror. The plot shows the power reflected back relative to the probes distance from the mirror. The peak power occurs at a focal distance of $0.8 \mathrm{~mm}$. The full width at half $\max$ (FWIM) corresponding to the depth of field was found to be $0.6222 \mathrm{~mm}$.

For a faster and more practical characterization during the probe manufacturing process, a probe was attached to a galvo scanner and placed over a titanium dioxide $\left(\mathrm{TiO}_{2}\right)$ phantom. The $\mathrm{TiO}_{2}$ phantom was chosen because it is a highly scattering medium with a relatively low absorption coefficient. The reference arm length of the interferometer was set so that the surface of the probe would appear just above the imaging window on the computer screen. The probe would then be positioned at a distance away from the $\mathrm{TiO}_{2}$ sample that would make the sample appear the brightest and that distance would be taken as the working distance. Knowing that the display shows a depth of $3 \mathrm{~mm}$, the focal length could then be calculated. This method was much faster and gave an imaging sample for each potential probe. A sample image used for this characterization method can be seen in Figure 2.23. 


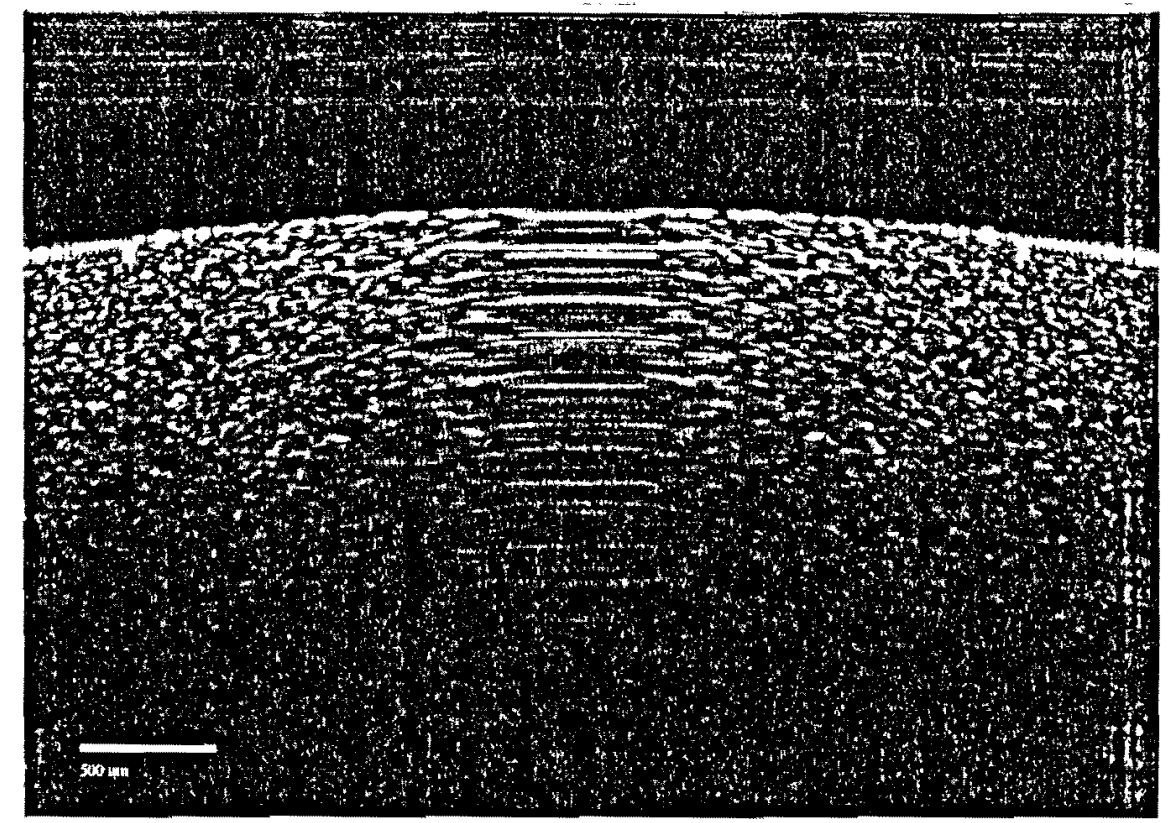

Figure 2.23 - Titanium dioxide phantom imaged to test the quality of a probe at the forward-looking stage. The streaks in the center of the image may have been caused by the galvo slowing down midway through its motion causing multiple A-scans over the same region of the phantom. The scale bar is $500 \mu \mathrm{m}$.

Various combinations of CL spacers and GRIN fibers were tested. Table 4 shows the results of these tests.

\begin{tabular}{|c|c|c|}
\hline CL Spacer $(\mathbf{m m})$ & GRIN $(\mathbf{m m})$ & Working Distance $(\mathbf{m m})$ \\
\hline 0.498 & 0.139 & 0.38 \\
\hline 0.459 & 0.173 & 0.43 \\
\hline 0.492 & 0.165 & 0.55 \\
\hline 0.479 & 0.144 & 0.94 \\
\hline 0.470 & 0.163 & 0.99 \\
\hline 0.476 & 0.175 & 1.31 \\
\hline 0.487 & 0.137 & 1.46 \\
\hline 0.472 & 0.143 & 1.48 \\
\hline 0.488 & 0.144 & 1.52 \\
\hline 0.485 & 0.143 & 1.90 \\
\hline 0.467 & 0.169 & 2.09 \\
\hline
\end{tabular}

Table 2.4 - Combinations of various CL spacer and GRIN lengths and the resulting working distance.

The probe used for the radial imaging presented from here on has the specs of the row italicized in Table $2.4(\mathrm{CL}$ Spacer $=0.470 \mathrm{~mm}, \mathrm{MM}=0.163 \mathrm{~mm}$, Working Distance $=$ 
$0.99 \mathrm{~mm}$ ). With an outer catheter outside diameter of $1 \mathrm{~mm}$, the focal point is formed at about $0.5 \mathrm{~mm}$ outside the catheter. The optics of this probe imaged at $100 \mathrm{x}$ magnification with a stereo microscope can be seen in Figure 2.24.

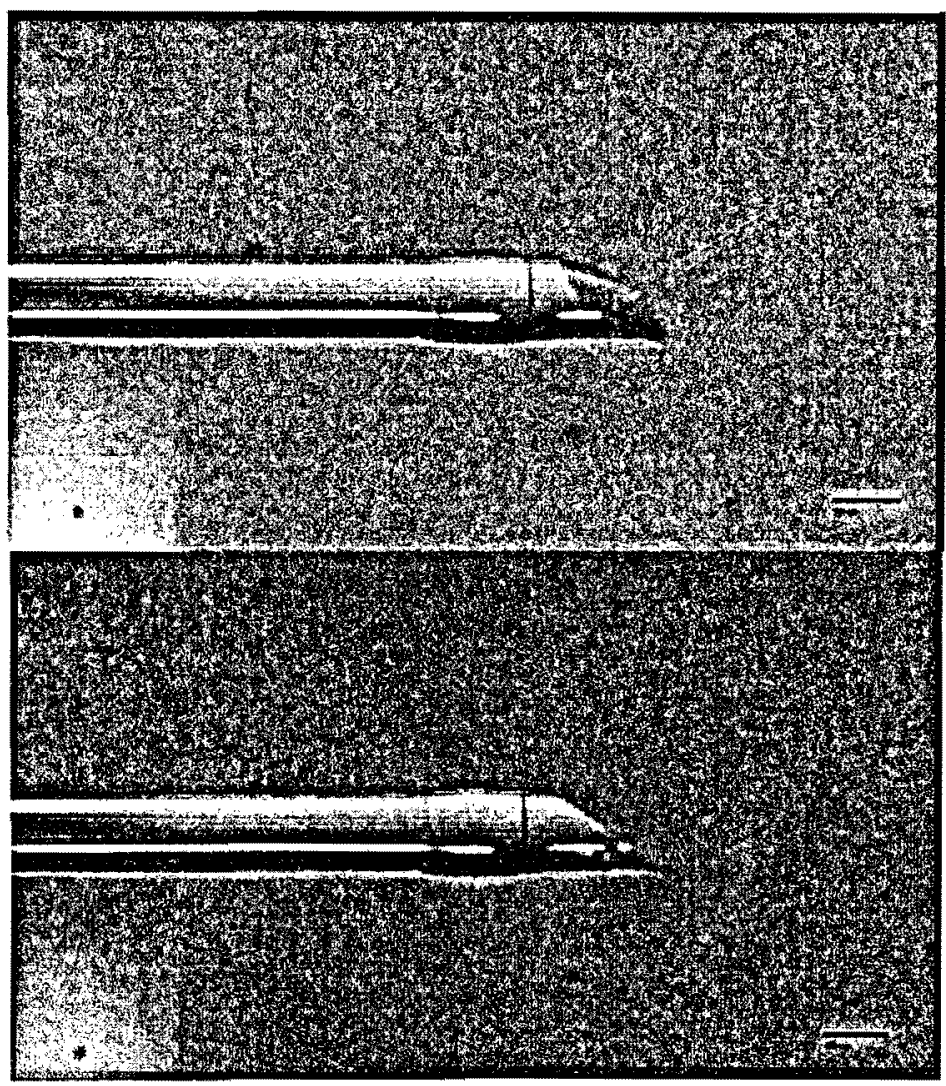

Figure 2.24 - Probe optics with CL spacer magnified 100x viewed from two different angles. The scale bars are $100 \mu \mathrm{m}$.

Figure 2.25 is an image taken with the probe taped to an IR card. It can clearly be seen that the longer working distance has resulted in a much better image with a better SNR ratio. 


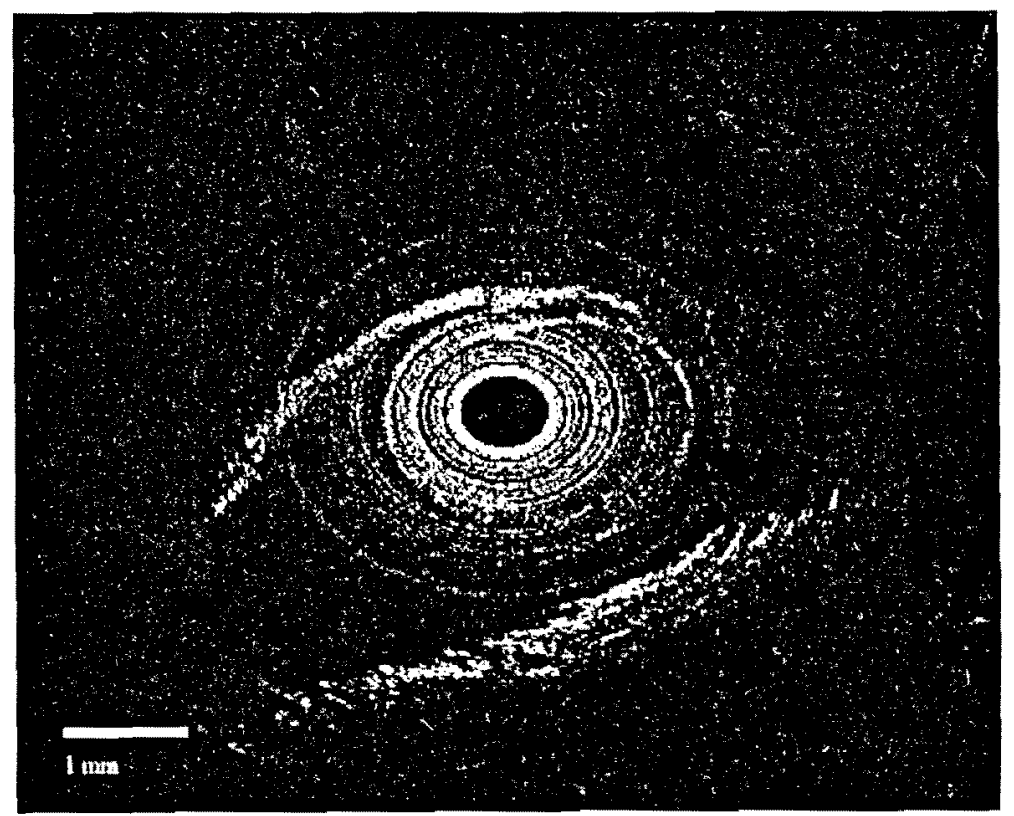

Figure 2.25 - Probe with CL Spacer taped to an IR card. 


\section{CHAPTER 3：3-D IMAGING}

This chapter will go through the image processing techniques used to create three dimensional images with the radial system. The outer ear of a mouse was imaged in vivo in order to test the image processing techniques.

The system used to acquire the raw data that corresponds to an image while scanning a sample is a system designed to acquire data from a raster scanning galvo probe. For this reason the data acquired during a radial scan had to go through some post processing. Before data is acquired with the system the number of A-scans that the user wants to have in their image is entered into the system. The system acquires data at $36000 \mathrm{~A}$-scans/second. For radial imaging, the A-scan acquisition rate is divided by the rotation rate to get the number of A-scans desired for a frame. For example, a $5 \mathrm{~Hz}$ rotation rate would require $7200 \mathrm{~A}$-scans/frame to obtain the full 360 degrees worth of data. The system sends a trigger signal at the beginning of each frame. After the data for a whole frame is acquired there is a lag in the system before the next trigger to acquire the next frames worth of data. This lag lasts anywhere from a few milliseconds to a few hundred milliseconds and is dependent on the load put on the system CPU. Since the load on a system always varies slightly, the lag time is always different. For radial imaging this lag can cause a severe misalignment between frames. In order to correct for this lag the A-scans in an image are shifted according the lag time caused by the previous frames. Once the images are aligned they are made radial. The A-scans from the original image are concentrically fanned around a center point to create a radial image. When the images are fanned out small gaps may appear between A-scans. To correct for this the A-scans from the original image are spread out horizontally and artificial A-scans are 
placed in between the original A-scans to fill in the gaps created. The artificial A-scans are interpolated from the adjacent originals. If only one artificial A-scan is placed in between 2 originals, the artificial A-scan is obtained by adding up the two originals and dividing them by 2 . If more than one artificial A-scan is required between two originals, a linear weighted averaging method is used based on which original the artificial A-scan is closer to. For example, if there are 3 artificial A-scans between 2 original A-scans (org1, art1, art2, art3, org2), then art1 would be equal to org1(.75) plus org2 (.25), art2 would be equal to org1(.5) plus org2 (.5), and art3 would be equal to org1(.25) plus org2 (.75). Once the radial images are created they are saved as 8-bit unassigned integers (uint8) and saved in a tif file format in order to be easily read by an imaging software called OsiriX [31]. The MATLAB code used for the post processing can be found in the Appendix.

The outer ear of an in vivo mouse was imaged. A mouse was borrowed from a group at the end of a window chamber study. The mouse was a healthy anesthetized mouse used for a proof of principle study where a patch of skin was removed on one side of the mouse's back in order to image the inside of a patch of skin on the opposite side. Figure 3.1 is an image of the mouse with the ear that was imaged. 


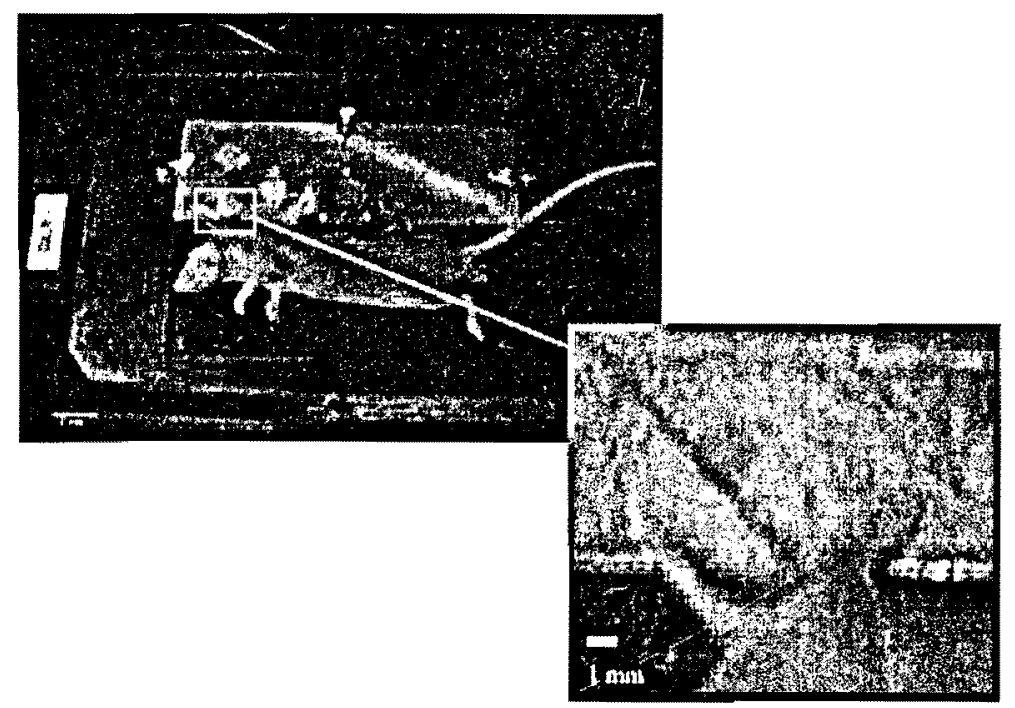

Figure 3.1 - Window chambered mouse with the imaged ear. The line in the ear image is a wire that the probe was placed next to during the radial imaging. The scale bar in the top left image is $1 \mathrm{~cm}$.

The radial probe was placed next to the wire in the image. The radial probe was spinning at $5 \mathrm{~Hz}$ and pulled back over a $6 \mathrm{~mm}$ region in 70.62 seconds. Due to the lag between frame acquisitions only 240 frames were acquired. Figure 3.2 shows the resulting 3-D image.

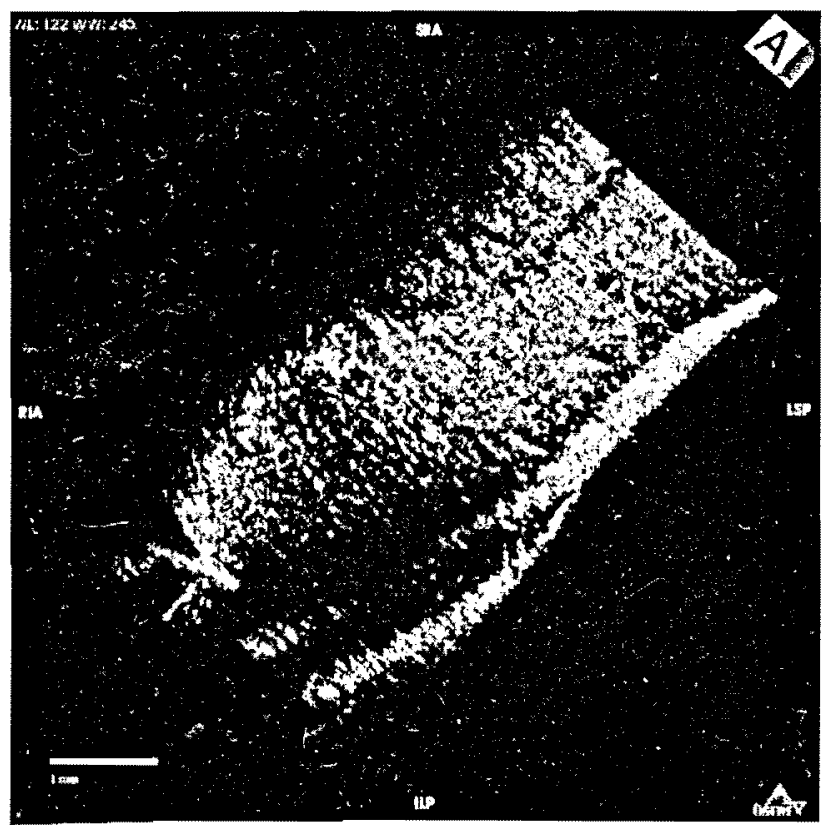

Figure 3.2-3-D image of a radial scan of a mouse ear. The scale bar is $1 \mathrm{~mm}$. 
The bright line on the right of the figure is the wire next the probe. The image is oriented such that the head of the mouse would be in the bottom left corner. Figures 3.3 and 3.4 are the axial and lateral cross sections from different locations within the sampled area. The image was volume rendered in order to create these cross sections. A section from one of the lateral cross sectional image was compared to histology and an OCT image taken with a galvo scanner over the same region of interest. This comparison can be seen in Figure 3.5. The histology image was adapted from [32].
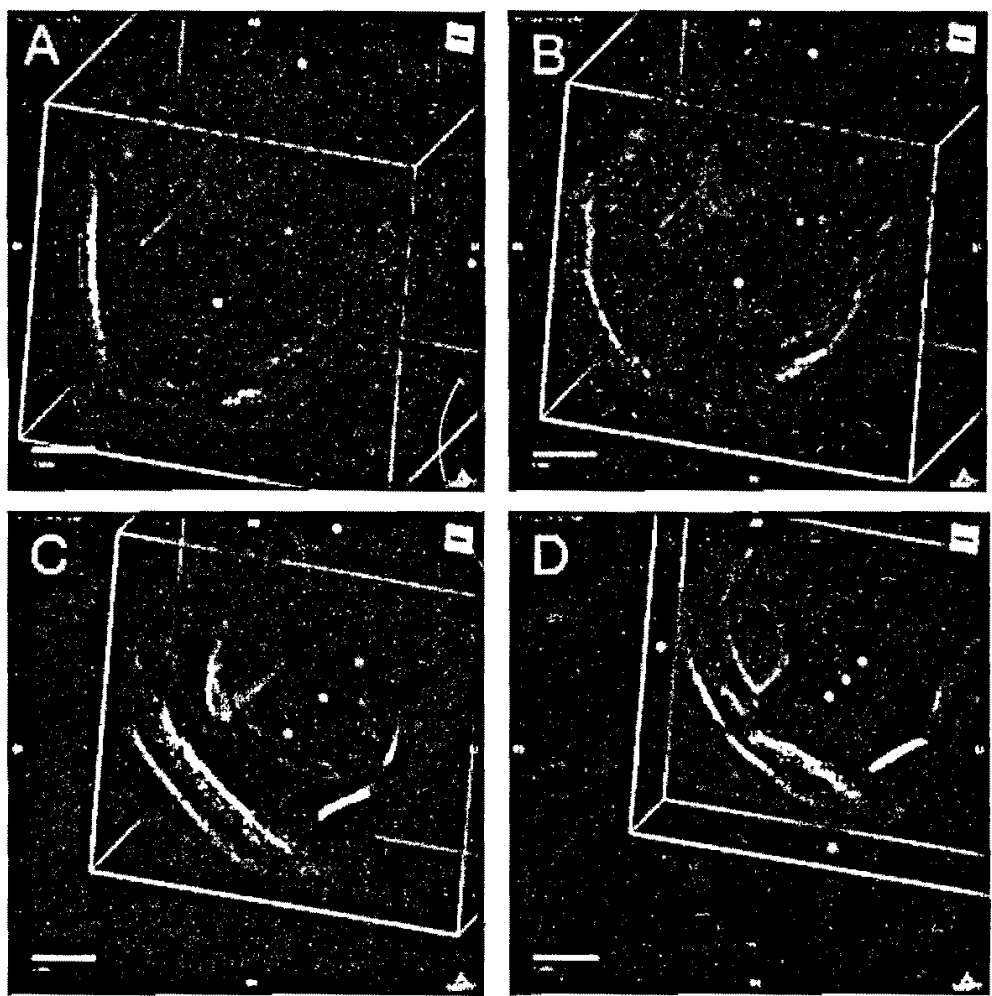

Figure 3.3 - Axial cross sections of the mouse ear image. The slices progress from $A$ to $D$ looking from the bottom left corner of Figure 3.2 and progressing towards the top right. The scale bars are $1 \mathrm{~mm}$. 

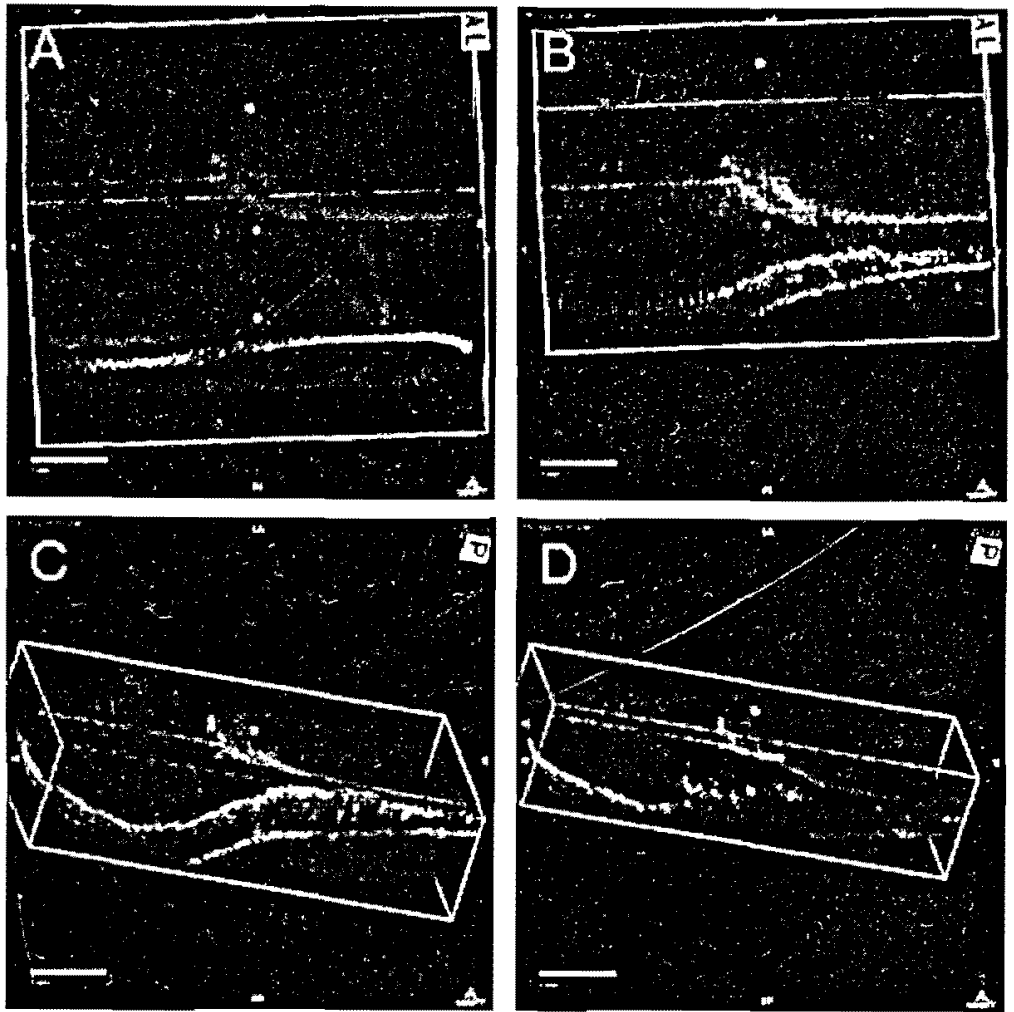

Figure 3.4 - Lateral cross sections of the mouse ear image. The slices progress from $A$ to $D$ looking from the bottom right corner of Figure 3.2 and progressing towards the top left. The scale bars are $1 \mathrm{~mm}$.

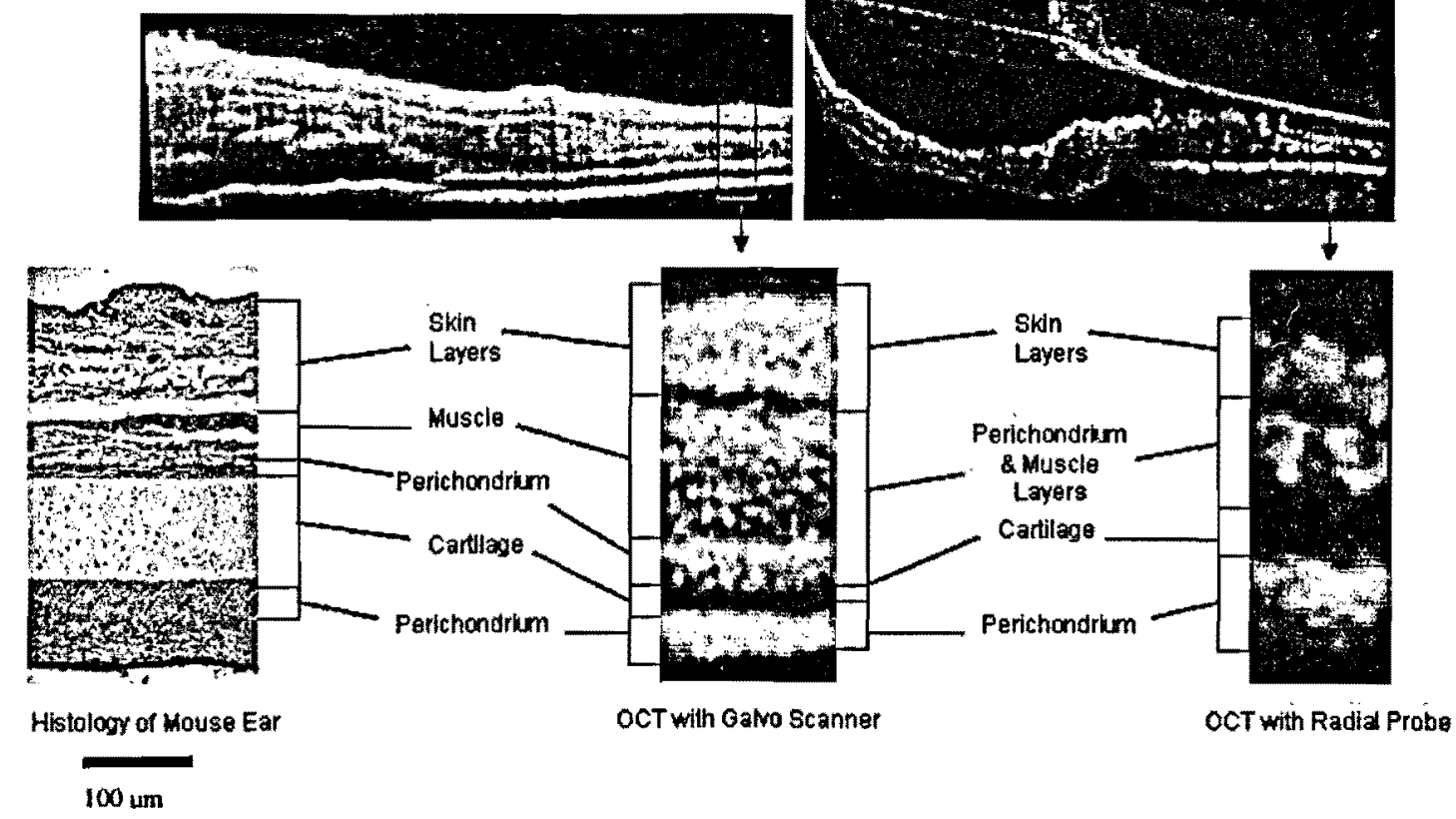

Figure 3.5 - Cross section of the mouse ear scan with a radial probe compared to the galvo scanner results and histology. The histology image was adapted from [32]. 
From Figure 3.5 it can be seen that the radial imaging was successful in identifying the general structures of the mouse ear. It was difficult to identify finer layers, such as the thin layer of perichondrium above the cartilage. This may be due to the fact that the radial image only had 240 slices with a thickness of 25 microns, where the galvo image had 1027 slices with a thickness of about 6 microns. Another contribution to the relatively poor resolution may be that the tissue was near the edges of the depth of field. These issues will need to be taken into consideration for future imaging. 


\section{CHAPTER 4: CONCLUSION}

\subsection{Summary}

The objective of this thesis project was to develop a rotational catheter based OCT imaging system capable of three dimensional imaging in vivo via trans-luminal methods. This objective was put forth in order to further the development of technology within the OCT group in Toronto and in turn increase the labs contribution to the medical imaging community with a wider range of studies made possible. The objective put forth was met and thus the hypothesis was confirmed. A working prototype of a rotational catheter based OCT imaging system capable of three dimensional imaging has been constructed. The system is capable of rotating at a rate of $200 \mathrm{~Hz}$. At this rate the rotary system will allow for a frame acquisition rate of $200 \mathrm{fps}$ which is significantly higher than the highest published acquisition rate to date of $108 \mathrm{fps}$. The probes used with the system were modeled after the Intravascular Ultrasound (IVUS) miniature torque cable design. The toque cable is a tightly wound stainless steel cable with an outer diameter of $0.7 \mathrm{~mm}$ that acts as a catheter that rotates with the optical fiber. The outermost catheter is a biocompatible USP Class VI teflon tube with an outer diameter of about $1 \mathrm{~mm}$. The probes can be sealed and sterilized between subjects without being damaged; unlike the single use IVUS probes. The optics on the fiber portion of the probe is comprised of an arrangement of CL and GRIN fibers. An optics design with a CL spacer was chosen because of its superior ability to create longer working distances. The probe created with this design had a working distance of $0.99 \mathrm{~mm}$ putting the focal point approximately .5 mm away from the outer catheter. The rotary probe was placed on the outer ear of an in vivo mouse. A rotary and pull back scan was performed over $6 \mathrm{~mm}$ of tissue with a 
rotational rate of $5 \mathrm{~Hz}$ and a three dimensional image was created from the data gathered. A portion of a lateral slice from the image was compared to the general histology of a mouse ear and the results of a galvo scan over the same region. The image compared well to the general anatomy found in the histology, but it did not have as high a resolution as the image taken with the galvo scanner. This may have been due to the fact that the image taken with the galvo scanner had 1027 slices over the $6 \mathrm{~mm}$ region and the radial scan only had 240 slices. Another contribution to the relatively poor axial resolution may have been that the tissue was near the edges of the depth of field. This is an issue that needs to be looked at more closely in the future. A working prototype of a rotational catheter based OCT imaging system capable of three dimensional imaging was constructed, thus meeting the main objective put forth at the beginning of the project.

\subsection{Future Work}

\subsubsection{Doppler}

The raw data from the mouse ear imaging was treated with the Kasai velocity estimation algorithm developed by the Toronto OCT group [6] in order to get a Doppler signal indicating blood flow. To increase the chances of finding a vessel the probe was placed directly above a vessel next to the wire that can be seen in Figure 3.1. Figure 4.1 shows a structural image with its corresponding Doppler image. 

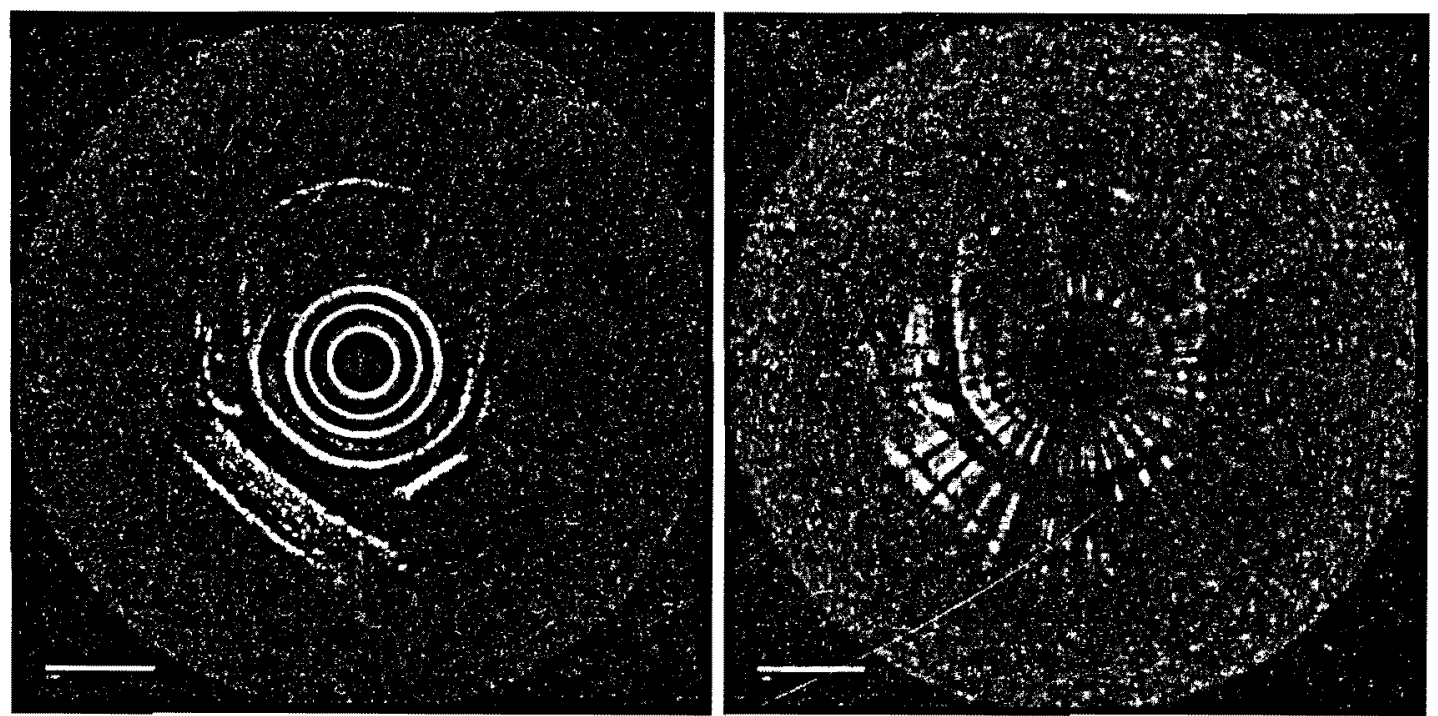

Figure 4.1 - Left: A structural frame of the mouse ear. Right: Corresponding Doppler image. The scale bars are $1 \mathrm{~mm}$.

With this Doppler image it is difficult to clearly locate a vessel or any moving tissue. The Doppler image gives the impression that there may have been some bulk tissue motion with the ear, but the ear was stationary for the duration of the scanning process. This is an indication that the probe was jittering while it rotated. This could have been caused by the probe not being centered properly within the outer catheter. Another issue with the Doppler image is noise. The flickering on the pixels caused by noise was misinterpreted by the Doppler code as a moving object. These issues need to be looked at more closely for future imaging with radial probes. The SNR needs to be improved and the axial streaking caused by the probe jitter needs to be dealt with. With regards to improving SNR, the FORJ needs to be replaced. The FORJ was the main cause of signal loss to and from the sample. Solutions to this problem may be developing an FORJ inhouse or working closely with other groups who have built an FORJ with a lower insertion loss. If an FORJ is developed in house, the fiber in the rotor and stator can be designed with a quarter pitch GRIN fiber stage at the alignment point in order to expand 
and collimate the beam at the stator end and receive a larger amount of light at the rotor end. The streaks caused by the probe jitter could be fixed by treating the images with a histogram rejection technique developed by the Toronto group [33]. The technique was developed to remove bulk tissue motion from the Doppler image, but it can be applied to remove the artifacts caused by the radial probe jitter. The technique identifies and removes the artifacts in an A-scan by creating a histogram of the like entries in an A-scan and removing the entries on the image that have the highest peak in the histogram. The entries with the highest peak will most likely be the entries caused by the jitter because the jitter affects the whole A-scan. Deleting the entries caused by the jitter will likely leave only the entries caused by fluid flows.

\subsubsection{Mouse Aorta Imaging}

With the trans-lumenary capabilities of the rotational catheter based system developed in this project, there are many possible future projects. Although, there are two projects that the author will be dealing with in the near future. One is a short term project and the other is more long term. The short term project is the trans-lumenary imaging of an ex vivo mouse aorta and the long term project is the in vivo transesophageal imaging of a mouse aorta. Work has already begun for the short term project. An ex vivo mouse aorta was imaged using a galvo scanner. The mice were bread to be susceptible to having arterial plaques. Figure 4.2 and 4.3 show the resulting three dimensional images. 


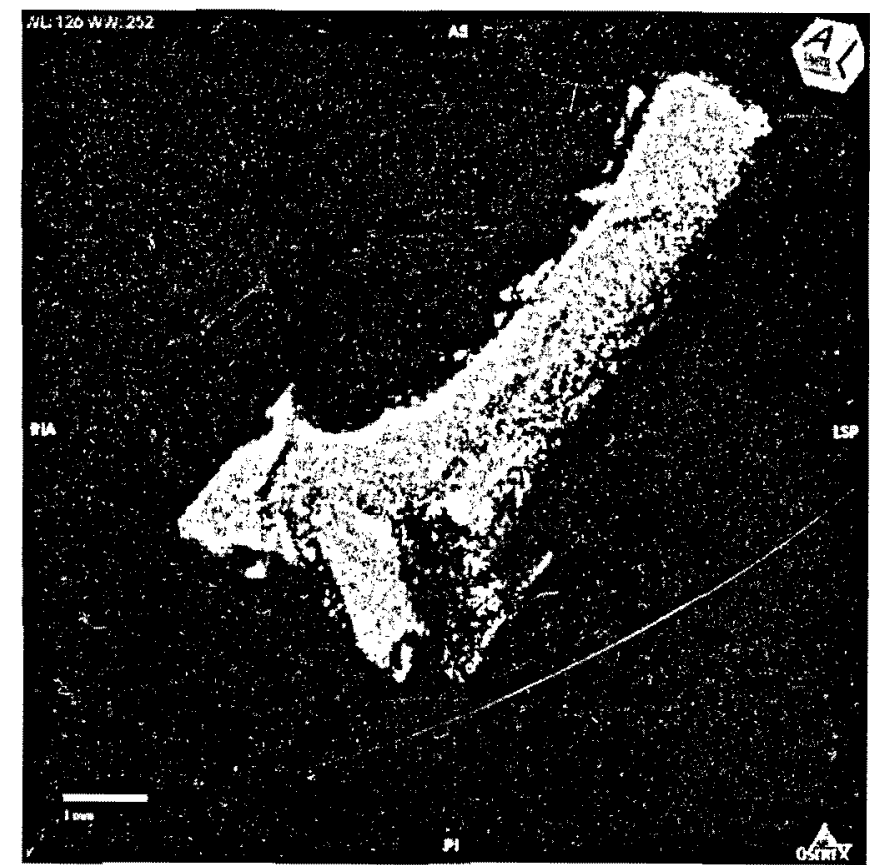

Figure 4.2 - Three dimensional SS-OCT image of an ex vivo mouse aorta. The long portion going from the top right to the bottom left is the descending aorta. The aortic arch is on the bottom left with bifurcations leading to the left subclavian artery (bottom right vessel), left common carotid artery (center vessel), and the brachiocephalic artery (bottom left vessel) that eventually leads to the right common carotid and right subclavian arteries (not shown).

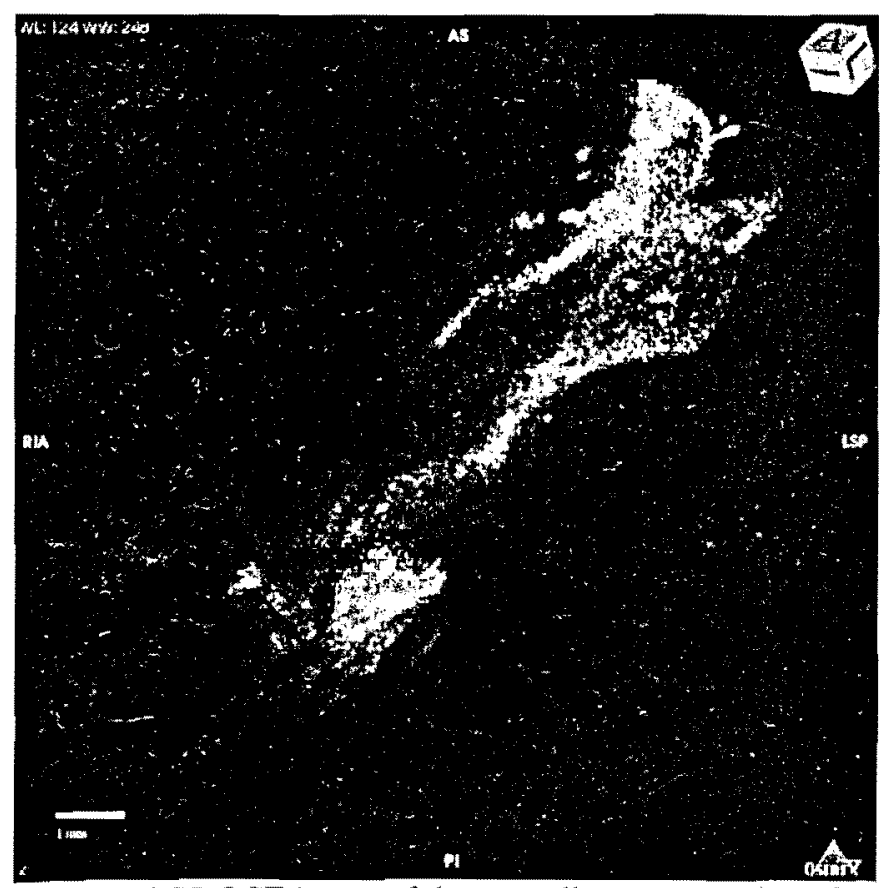

Figure 4.3 - Three dimensional SS-OCT image of the ascending aorta portion of an $e x$ vivo mouse aorta. The long portion going from the bottom left to the top right is the ascending aorta. The top right has the bifurcation of the ascending aorta to the aortic arch (turning to the right) and the brachiocephalic artery (continuing to the top right). 
The axial and lateral cross sections of these images led to some definitive plaque discoveries indicated by the irregularities in the aortic wall surface. Some of the cross sections are shown in Figures 4.4, 4.5, and 4.6.

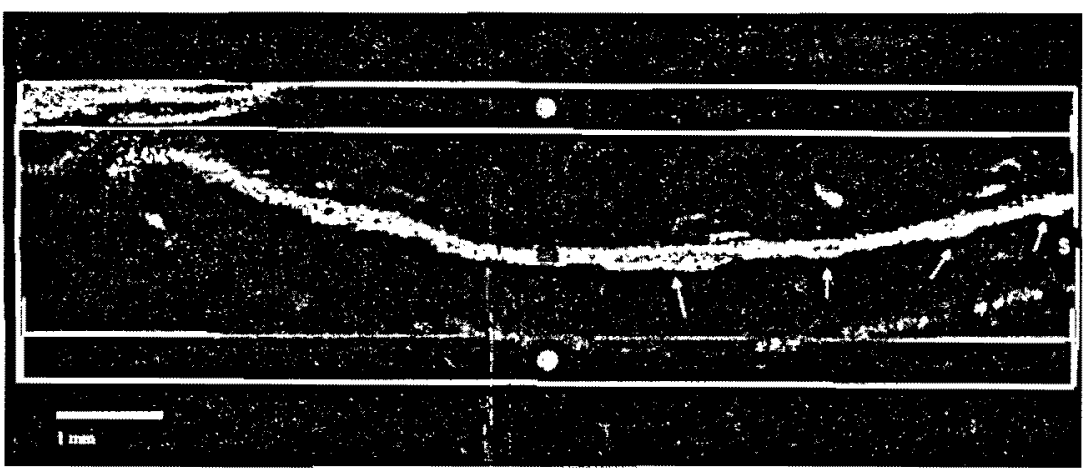

Figure 4.4 - Lateral cross section of the descending aorta portion of Figure 4.2. The arrows indicate the location of some plaques
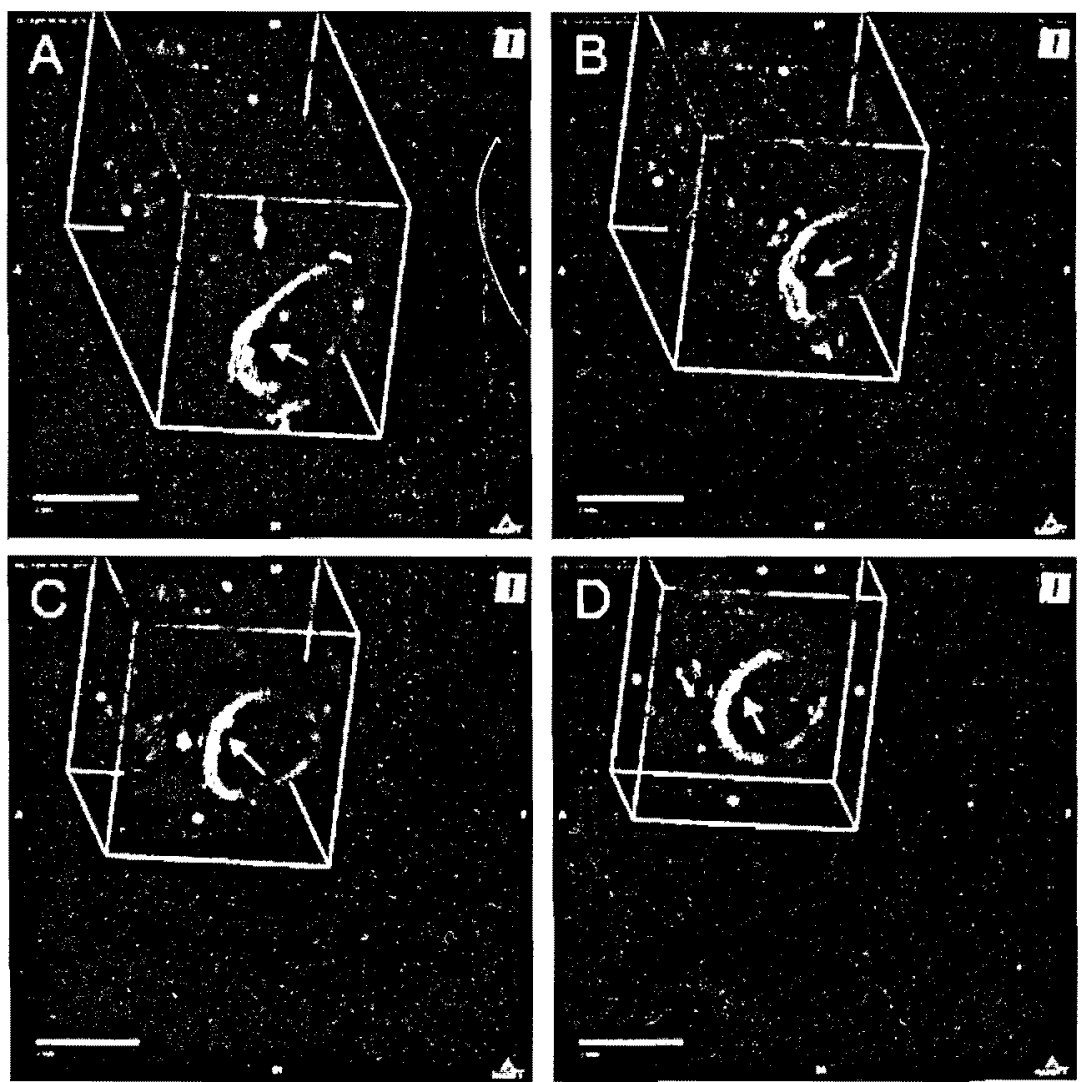

Figure 4.5 - Axial cross sections of the mouse aorta. The slices progress from A to D looking from the bottom left corner of Figure 4.2 just past the aortic arch (beginning of the descending aorta) and progressing towards the top right. The arrows indicate the location of some plaques 

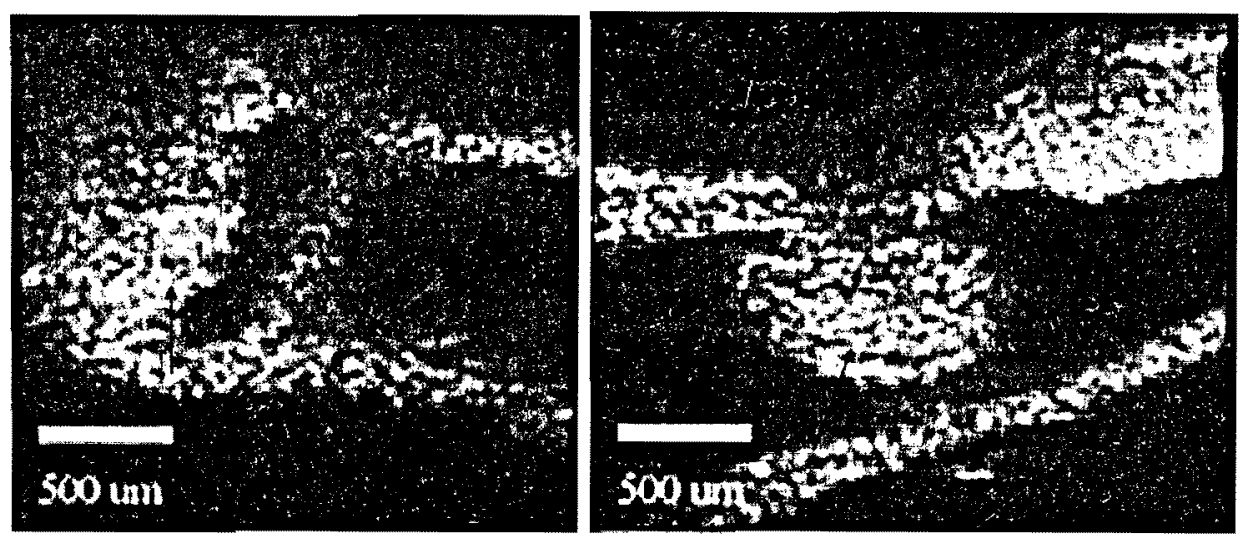

Figure 4.6 - Left: Axial cross section of the bifurcation of the ascending aorta from Figure 4.3 with the aortic arch on the right and the brachiocephalic artery on the left. Right: Lateral cross section of the brachiocephalic artery. A large plaque (indicated by the arrows) is evident in the brachiocephalic artery.

In Figures $4.4,4.5$, and 4.6 it is evident by the irregularities in the cross sections that SSOCT can identify the location of plaques in a mouse aorta. In these images it is also evident that it is difficult to differentiate between the plaques and the aortic wall with these galvo scan images. An ultra thin radial probe is being built in order to perform a trans-lumenary image of a mouse aorta in hopes of being able to differentiate between the plaque and the aortic wall (much like in Figure 1.9).

The more long term project will be the in vivo trans-esophageal imaging of a mouse aorta. The project will build on the work previously done by the Toronto OCT group with trans-esophageal imaging. Figure 4.7 is a two dimensional trans-esophageal Doppler OCT image of a rat in vivo. This figure shows the dorsal wall motion and the blood flow velocity associated with the aortic blood flow of a rat. 


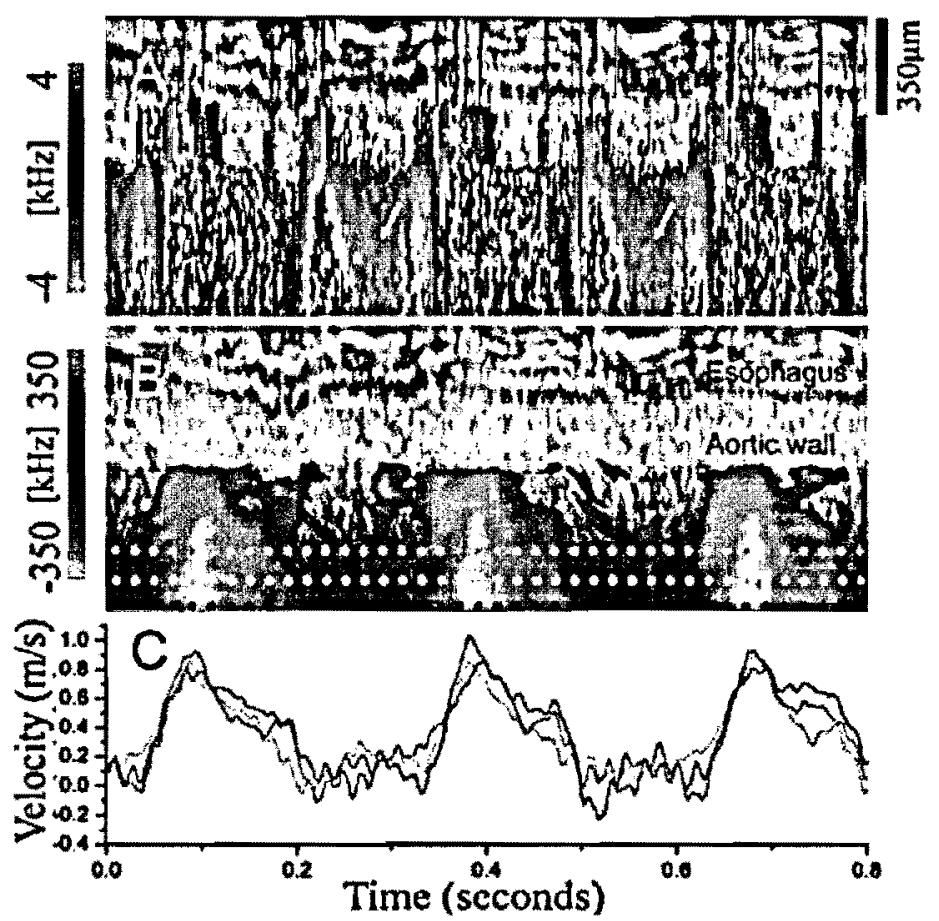

Figure 4.7 - In vivo images of aortic blood flow in a rat using transesophageal Doppler OCT methods. A) Doppler signals indicating aortic wall motion. The white arrows show regions of slow flow between heart beats. B) Image A) treated with an axial Kasai Algorithm. This accentuates the high speed blood flow regions. C) Temporal flow profiles measured at the dotted lines in B). [6]

The objective will be to obtain the type of results in Figure 4.7 in four dimensions with a radial pullback scan. The two issues with obtaining a Doppler image with the rotary system mentioned earlier need to be dealt with before this type of trans-esophageal imaging can be attempted. 


\section{REFERENCES}

[1] David Huang, Eric A. Swanson, Charles P. Lin, Joel S. Schuman, William G. Stinson, Warren Chang, Michael R. Hee, Thomas Flotte, Kenton Gregory, Carmen A. Puilafito, and James G. Fujimoto, "Optical Coherence Tomography", Science 254(5035), 1178 (1991)

[2] Morten Fruelund, "Development of Intravascular Catheter Probe for OCT Imaging of Vulnerable Plaques", MSc. Thesis, Graduate Department of Engineering, University of Southern Denmark (2005)

[3] Victor Yang, "Endoscopic Doppler Optical Coherence Tomography", PhD. Thesis, Department of Medical Biophysics, University of Toronto (2004)

[4] Adrian Mariampillai, Beau A. Standish, Nigel R. Munce, Cristina Randall, George Liu, James Y. Jiang, Alex E. Cable, I. Alex Vitkin, and Victor X.D. Yang, "Doppler Optical Cardiogram Gated 2D Color Flow Imaging at $1000 \mathrm{fps}$ and $4 \mathrm{D}$ in vivo Visualization of Embryonic Heart at 45 fps on a Swept Source OCT System", Optics Express 15(4), 1627-1638 (2007)

[5] Heng Li, Beau A. Standish, Adrian Mariampillai, Nigel R. Munce, Youxin Mao, Stephanie Chiu, Norman E. Marcon, Brian C. Wilson, Alex Vitkin, and Victor X.D. Yang, "Feasibility of Interstitial Doppler Optical Coherence Tomography for In Vivo Detection of Microvascular Changes During Photodynamic Therapy", Lasers in Surgery and Medicine 38, 754-761 (2006)

[6] Darren Morofke, Michael C. Kolios, Alex Vitkin, and Victor X. D. Yang, "Wide Dynamic Range Detection of Bidirectional Flow in Doppler Optical Coherence Tomography using a Two-dimensional Kasai Estimator", Optics Letters 32(3), 253255 (2007)

[7] Benjamin J. Vakoc, Milen Shishko, Seok H. Yun, Wang-Yuhl Oh, Melissa J. Suter, Adrien E. Desjardins, John A. Evans, Norman S. Nishioka, Guillermo J. Tearney, and Brett E. Bouma, "Comprehensive Esophageal Microscopy by using Optical Frequency-Domain Imaging", Gastrointestinal Endoscopy 65(6), 898-905 (2007)

[8] M. Samir Jafri, Suzanne Farhang, Rebecca S. Tang, Naman Desai, Paul S. Fishman, Robert G. Rohwer, and Cha-Min Tang, "Optical coherence tomography in the diagnosis and treatment of neurological disorders", Journal of Biomedical Optics 10(5), $051603(2005)$

[9] Uma Maheswari Rajagopalan and Manabu Tanifuji, "Functional optical coherence tomography reveals localized layer-specific activations in cat primary visual cortex in vivo", Optics Letters 32(17), 2614-2616 (2007) 
[10] Wolfgang Drexler and James G. Fujimoto, "State-of-the-art Retinal Optical Coherence Tomography", Progress in Retinal and Eye Research 27, 45-88 (2008)

[11] Adam M. Zysk, Freddy T. Nguyen, Amy L. Oldenburg, Daniel L. Marks, and Stephen A. Boppart, "Optical coherence tomography: a review of clinical development from bench to bedside", Journal of Biomedical Optics 12(5), 051403 (2007)

[12] S. H. Yun, G. J. Tearney, J. F. de Boer, N. Iftimia and B. E. Bouma, "High-speed Optical Frequency-domain Imaging", Optics Express 11(22), 2953-2963 (2003)

[13] Mark Brezinski, "Optical Coherence Tomography: Principles and Applications", Amsterdam: Elsevier Inc. (2006)

[14] A. F. Fercher, C. K. Hitzenberger, G. Kamp, S. Y. Elzaiat "Measurement of intraocluardistances by backscattering spectral interferometry", Journal of Optical Communications 117 (1-2), 43-48 (1995)

[15] M. Wojtokowski, A Kowalczyk, R. Leitgeb, A. F. Fercher, "Full range complex spectral optical coherence tomography technique in eye imaging", Optics Letters 27(16), 1415-1417 (2002)

[16] Adrian Mariampillai, Beau A. Standish, Eduardo H. Moriyama, Mamta Khurana, Nigel R. Munce, Michael K. K. Leung, James Jiang, Alex Cable, Brian C. Wilson, Alex Vitkin, and Victor X. D. Yang, "Speckle Variance Detection of Microvasculature using Swept-Source Optical Coherence Tomography", Optics Letters 33(13), 1530-1532 (2008)

[17] Adam M. Zysk, Freddy T. Nguyen, Amy L. Oldenburg, Daniel L. Marks, and Stephen A. Boppart, "Optical coherence tomography: a review of clinical development from bench to bedside", Journal of Biomedical Optics 12(5), 051403 (2007)

[18] I. K. Jang, B. E. Bouma, D. H. Kang, S. J. Park, S. W. Park, K. B. Seung, K. B. Choi, M. Shishkov, K. Schlendorf, E. Pomerantsev, S. L. Houser, H. T. Aretz, and G. J. Tearney, "Visualization of coronary atherosclerotic plaques in patients using optical coherence tomography: comparison with intravascular ultrasound", Journal of the American College of Cardiology 39(4), 604-609 (2002)

[19] http://www.lightlabimaging.com/intl/prodsrv/catani.htm\# 
[20] Seok H Yun, Guillermo J Tearney, Benjamin J Vakoc, Milen Shishkov, Wang Y Oh, Adrien E Desjardins, Melissa J Suter, Raymond C Chan, John A Evans, IkKyung Jang, Norman S Nishioka, Johannes $F$ de Boer, and Brett $E$ Bouma, "Comprehensive volumetric optical microscopy in vivo", Nature Medicine 12(12), 1429-1433 (2006)

[21] http://www.princetel.com

[22] http://www.thorlabs.com

[23] http://www.ofr.com/tech_fiber_formula_1.htm

[24] http://www.parkermotion.com

[25] http://www.newport.com

[26] http://www.zaber.com

[27] http://www.bostonscientific.com

[28] http://www.smallparts.com/

[29] Youxin Mao, Shoude Chang, Sherif Sherif, and Costel Flueraru, "Graded-index fiber lens proposed for ultrasmall probes used in biomedical imaging", Applied Optics 46(23), 5887-5894 (2007)

[30] http://www.veeco.com/

[31] http://www.osirix-viewer.com/

[32] http://www.lltech.fr/images

[33] Victor X. D. Yang, Maggie L. Gordon, Alvin Mok, Youghua Zhao, Zhongping Chen, Richard S. C. Cobbold, Brian C. Wilson, Alex Vitkin, "Improved phaseresolved optical Doppler tomography using the Kasai velocity estimator and histogram segmentation", Optics Communications 208, 209-214 (2002) 
$X=$ runcode 3 (OCT_Eringe_2D);

$[\mathrm{X}$, Shift $]=$ aligñTime $(\mathrm{X}, \mathrm{x}$ _previous, Shift $) ;$

\% ------ Spreaded/Fanned Image --.--- $\frac{}{3}$

\&nlarge img by spreading A scans horizontally \& interpolating between horizontally

disp(" ... Spreading Image');

spreadedImg $=\operatorname{spreadInterp}(X) ;$

: Take A Scans and fan out along a circle by mappying each pixel

disp (' ...Fanning Image');

fannedImg = fanImage (spreadedImg);

: - - - Interpolated Image -...- $\frac{o}{8}$

o Interpolate new image line by line horizontally

disp(' ... Interpolating Image");

interpImg = interpFannedImg (fannedImg);

fname = strcat (file_name, num2str(frame_start+AddThisToFile), '. Lif');

\&Creates the file name

: Manipulation of the data to fit well into a tiff

$\mathrm{X} 2$ = interpImg. * 4

$\mathrm{X} 2=(\mathrm{X} 2+\mathrm{pi}) * 40 ;$

$\mathrm{x} 2=\mathrm{uint8}(\mathrm{x} 2)$;

Uses the doppler color map, and the grayscale image, to make an

\% RGB image, to fit well for tiff

$\mathrm{X} 2$ = grs2rgb (X2, doppler);

imwrite (X2, fname)

clear $X$ spreadetaIng fannedimg interpImg $x 2$

disp(sprintf ('....Done Frame: $q d$ ', index));

end

end 